\title{
Chiral Bis- $\pi$-allylpalladium Complex Catalyzed Asymmetric Allylation of Imines: Enhancement of Enantioselectivity and Chemical Yield in the Presence of Water
}

\author{
Rodney A. Fernandes, Anton Stimac, and Yoshinori Yamamoto* \\ Department of Chemistry, Graduate School of Science, Tohoku University, \\ Sendai 980-8578, Japan
}

\section{Supporting Information}

(32 pages)

General Information. Commercially available imines were procured from Aldrich Chemical Co.; while most were prepared by refluxing equimolar amount of corresponding aldehyde and amine in toluene or benzene with Dean-Stark removal of water. They were then purified by either high vacuum distillation or recrystallization. Most commercially supplied chemicals were freshly distilled or recrystallized before use. Solvents were dried by standard procedures. Dry THF refers to commercial dehydrated THF procured from Kanto Chem. Co., Inc, Japan. ${ }^{1} \mathrm{H}$ and ${ }^{13} \mathrm{C}$ NMR spectra were recorded on a Jeol JNM LA-300 spectrometer. The chemical shifts are reported in $\delta$ units relative to internal tetramethylsilane for ${ }^{1} \mathrm{H}$ NMR and $\mathrm{CDCl}_{3}$ was used as internal standard $(\delta=77.0)$ for ${ }^{13}$ C NMR. IR spectra were recorded on a Shimadzu FTIR-8200 A spectrometer. Mass spectra were recorded on Jeol JMS-HX 110 in EI mode. Optical rotations were measured on a Jasco DIP-1000 digital polarimeter. HPLC was performed on Shimadzu LC9A using Daicel CHIRALCEL OD or CHIRALCEL OD-R columns $(0.46 \mathrm{~cm} x$ $25 \mathrm{~cm}$ ). The absolute configuration was determined by converting amine 13a into 1-phenylbutylamine which exhibited optical rotation with + value. The literature value of $(S)-1$-phenylbutylamine is $[\alpha]_{\mathrm{D}}-21.3$ (c 1.3 , $\left.\mathrm{CHCl}_{3}\right)^{1}$. Hence the absolute configuration of 13a is confirmed to be $(R)$. Thus, by analogy the homoallylamines obtained are assumed to have $(R)$ configuration $^{2}$ (unless otherwise indicated).

\section{Preparation of the catalyst, 2a}

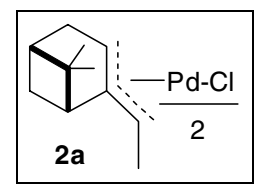

$(1 R)-(+)$-nopinone is available in Aldrich Chemical Co. in $90 \%$ ee. But its precursor $(1 S)-(-)-\beta$-pinene is available in $97 \%$ ee. So we preferred to prepare $(1 R)-(+)$-nopinone from $(1 S)-(-)-\beta$-pinene. The ozonolysis of exocyclic double bond of $(1 S)-(-)-\beta$-pinene by known procedure ${ }^{3}$ afforded $(1 R)-(+)$-nopinone in $75 \%$ yield, $[\alpha]_{\mathrm{D}}{ }^{22}+39.4(\mathrm{c}=1, \mathrm{MeOH})$. Wittig reaction with ethyltriphenylphosphonium bromide and ${ }^{t} \mathrm{BuOK}$ by known procedure $^{4}$ gave the olefin $7(E: Z=1: 1)$ in $83 \%$ yield. 


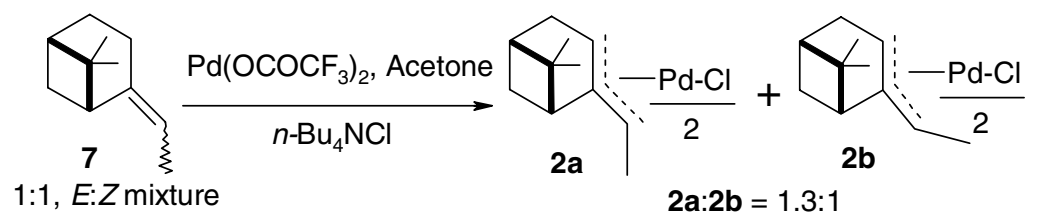

From olefin 7 we followed the literature procedure ${ }^{5}$ to prepare the $\mathbf{2 a}: \mathbf{2} \mathbf{b}$ mixture. To a solution of $\mathrm{Pd}\left(\mathrm{OCOCF}_{3}\right)_{2}$ $(5 \mathrm{~g}, 15.04 \mathrm{mmol})$ in dry acetone $(125 \mathrm{~mL})$ in a two necked round bottom flask under argon atmosphere was added olefin $7(2.26 \mathrm{~g}, 15.04 \mathrm{mmol})$. The reaction mixture was stirred for $1 \mathrm{~h}$ at room temperature (TLC monitored). ${ }^{n} \mathrm{Bu}_{4} \mathrm{NCl}(4.56 \mathrm{~g}, 16.55 \mathrm{mmol})$ in acetone $(30 \mathrm{~mL})$ was added and the reaction mixture was stirred for $1 \mathrm{~h}$. The clear brown-orange solution was then filtered through a plug of celite to remove suspended Pd-black. The filtrate was concentrated to thick amber colored oil. This was purified by silica gel column chromatography using hexane/EtOAc $=5: 1$ as eluent to give $\mathbf{2 a} / \mathbf{2 b}$ mixture as semi solid $(6.92 \mathrm{~g}) .{ }^{1} \mathrm{H}$ NMR indicated $\mathbf{2 a} \mathbf{2} \mathbf{2} \mathbf{b}=1.3$ : 1. This was dissolved in minimum $\mathrm{CH}_{2} \mathrm{Cl}_{2}$ and hexane $(30 \mathrm{~mL})$ was added. The crystallized solid was filtered through sintered funnel, washed with hexane and dried with suction to afford $\mathbf{2 a}: \mathbf{2} \mathbf{b}=4.8: 1$ (3.2 g) as yellow powder (I). Further upgradation is as shown in the flow chart below (II-V). During propionitrile recrystallization boiling of solvent to dissolve the solid should be avoided to prevent catalyst decomposition. To recover the catalyst the filtrates after each recrystallization F1-F3 as shown below were combined and concentrated to yellow colored solid foam. This was subjected to benzene column chromatography. Elution with benzene gave in first few fractions the catalyst $2 \mathbf{b}(0.45 \mathrm{~g})$ having $[\alpha]_{\mathrm{D}}^{22}+47.5\left(\mathrm{c}=0.4, \mathrm{CHCl}_{3}\right)$. The later fractions were mixture of $2 \mathbf{a}$ and $\mathbf{2 b}$. The filtrates $\mathbf{F} 4$ and $\mathbf{F 5}$ were combined and concentrated to yellow powder $(1 \mathrm{~g})$. This on successive three recrystallizations in propionitrile gave $\mathbf{2 a : 2 b}=100: 1(0.452 \mathrm{~g}),[\alpha]_{\mathrm{D}}{ }^{22}-17.2\left(\mathrm{c}=0.4, \mathrm{CHCl}_{3}\right)$. Further three repeated recrystallizations in propionitrile afforded $\mathbf{2 a}: 2 \mathbf{b}=>400: 1(0.215 \mathrm{~g}),[\alpha]_{\mathrm{D}}{ }^{22}-19.8(\mathrm{c}=0.4$, $\left.\mathrm{CHCl}_{3}\right)$. 


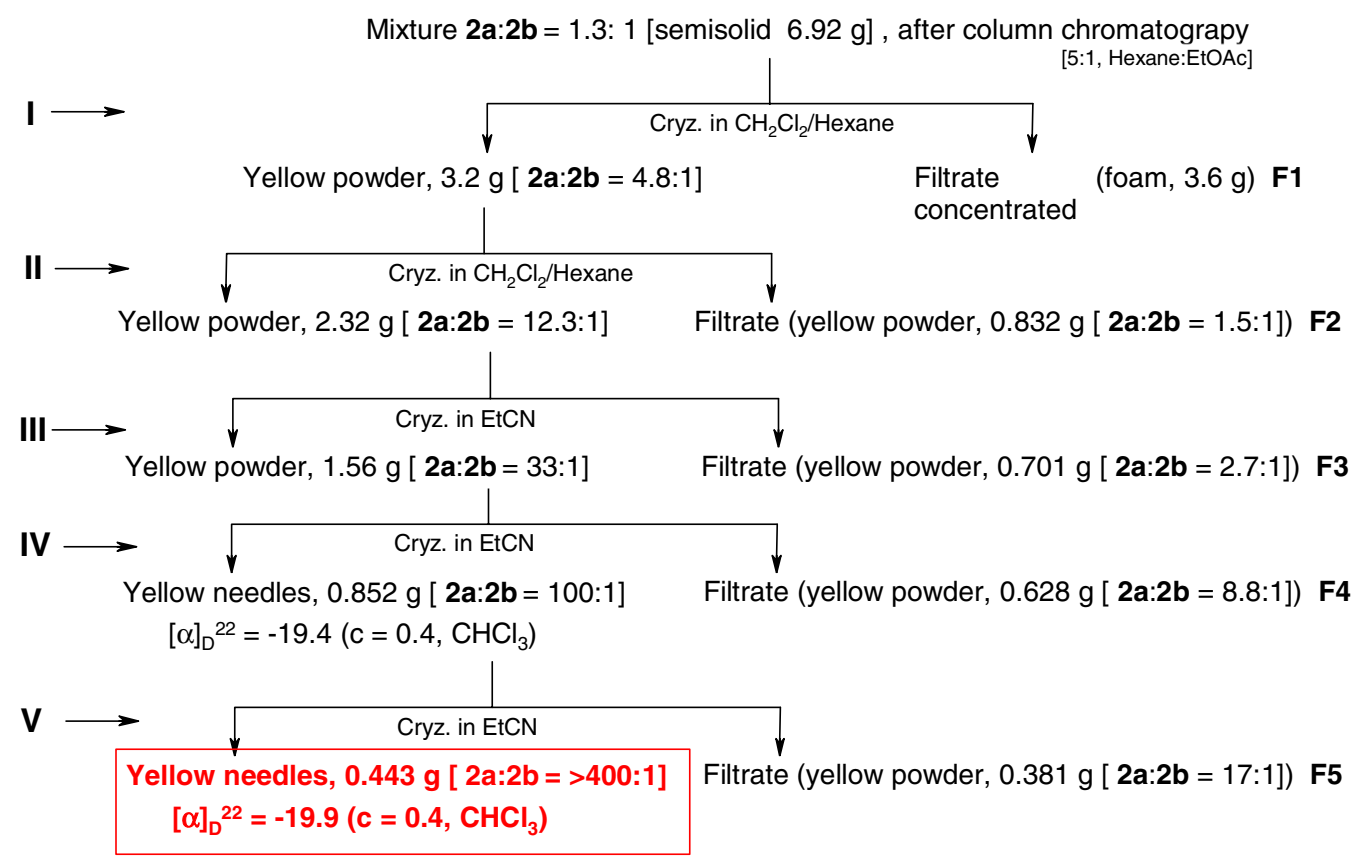

\section{Recovery of the catalyst}

Filtrates F1 - F3 $\longrightarrow$ Combined and subjected to benzene column separation 2b ligand was separated $(0.45 \mathrm{~g}),[\alpha]_{D}{ }^{22}+47.5\left(\mathrm{c}=0.4, \mathrm{CHCl}_{3}\right)$

Filtrates F4 and F5 $[1.0 \mathrm{~g}]$ Three repeated recrystallizations in EtCN

Yellow needles, $0.452 \mathrm{~g}$ [2a:2b $=100: 1]$

$[\alpha]_{D}^{22}=-17.2\left(\mathrm{c}=0.4, \mathrm{CHCl}_{3}\right)$

Three repeated

recrystallizations in EtCN

Yellow needles, $0.215 \mathrm{~g}[2 \mathrm{a}: 2 \mathrm{~b}=>400: 1]$

$[\alpha]_{D}^{22}=-19.8\left(\mathrm{c}=0.4, \mathrm{CHCl}_{3}\right)$

${ }^{1} \mathrm{H}$ NMR of 2a:2b = 1.3:1 


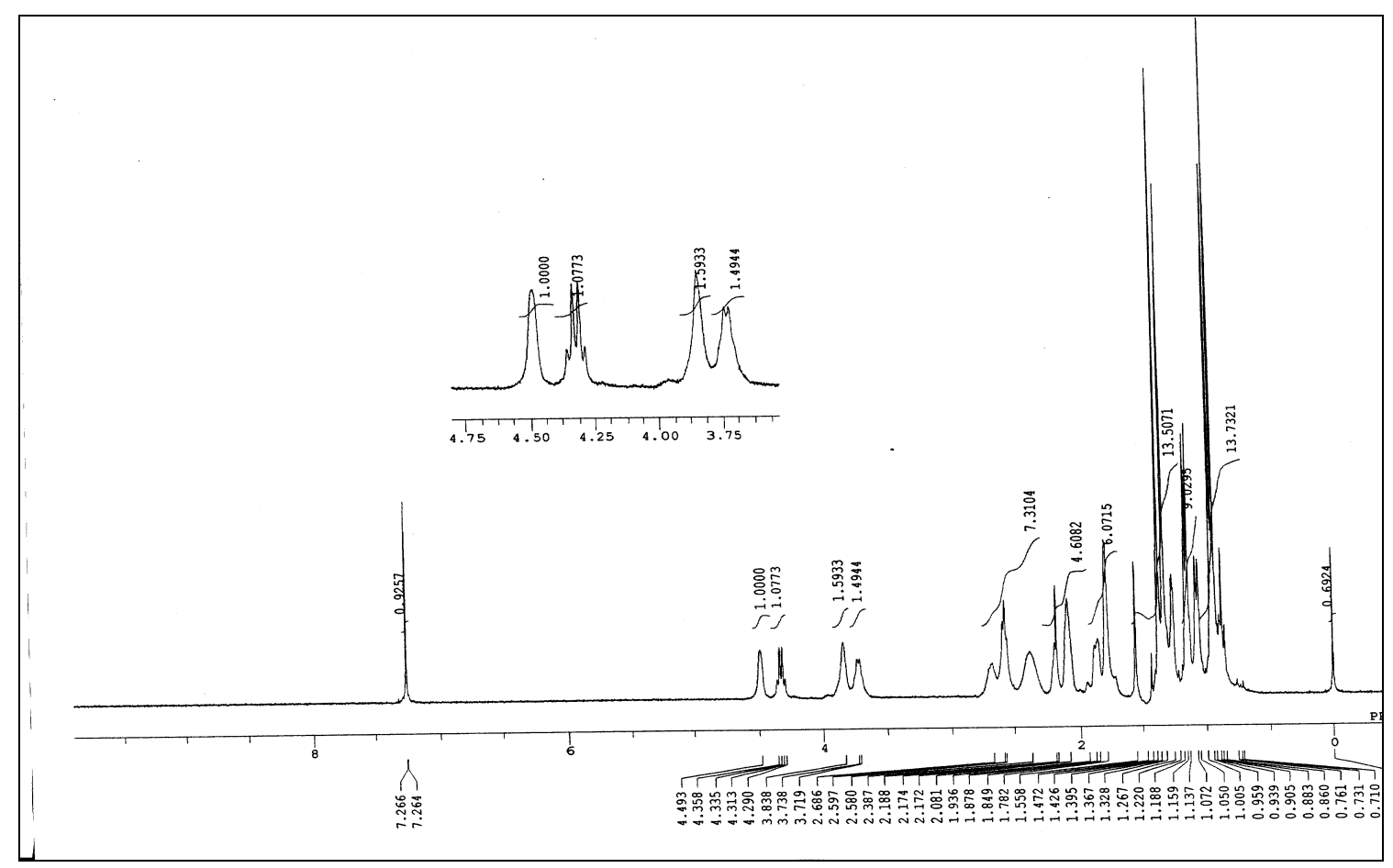

${ }^{1} \mathrm{H}$ NMR of 2a:2b = 100:1 


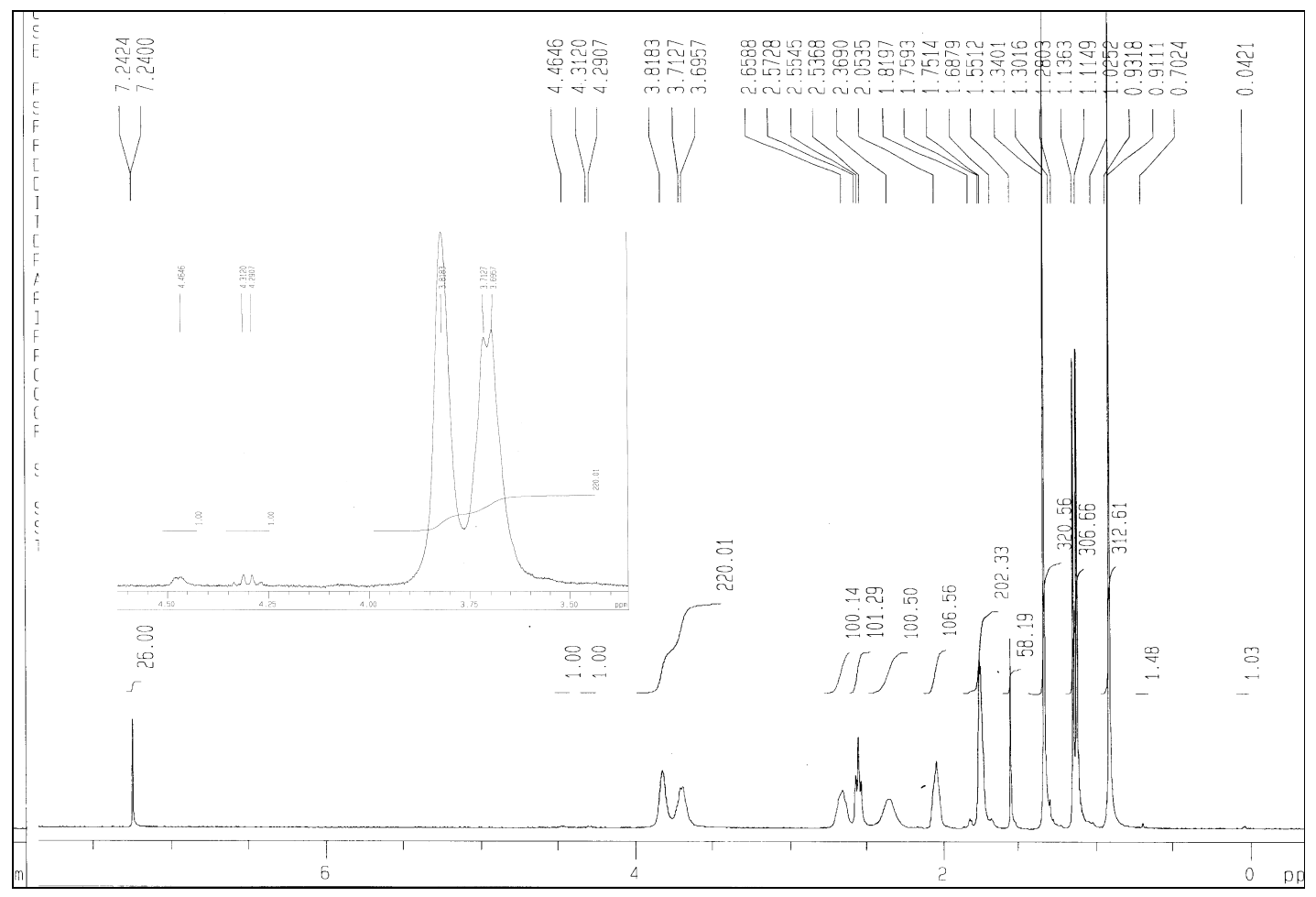

${ }^{1} \mathrm{H}$ NMR of $2 \mathrm{a}: 2 \mathrm{~b}=>400: 1$

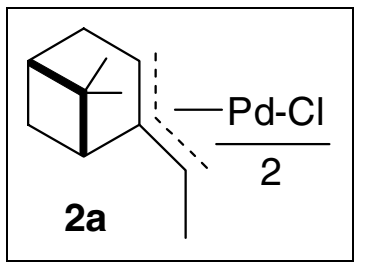

2a: $\left(\mathrm{CDCl}_{3}\right) \delta 3.84-3.80(\mathrm{~m}, 1 \mathrm{H}), 3.78-3.68(\mathrm{~m}, 1 \mathrm{H}), 2.7-2.64(\mathrm{~m}, 1 \mathrm{H}), 2.55(\mathrm{brt}, J=5.5 \mathrm{~Hz}, 1 \mathrm{H}), 2.44-2.33(\mathrm{~m}$, $1 \mathrm{H}), 2.08-2.03(\mathrm{~m}, 1 \mathrm{H}), 1.77-1.74(\mathrm{~m}, 2 \mathrm{H}), 1.34(\mathrm{~s}, 3 \mathrm{H}), 1.13(\mathrm{~d}, J=6.5 \mathrm{~Hz}, 3 \mathrm{H}), 0.91(\mathrm{~s}, 3 \mathrm{H})$. 


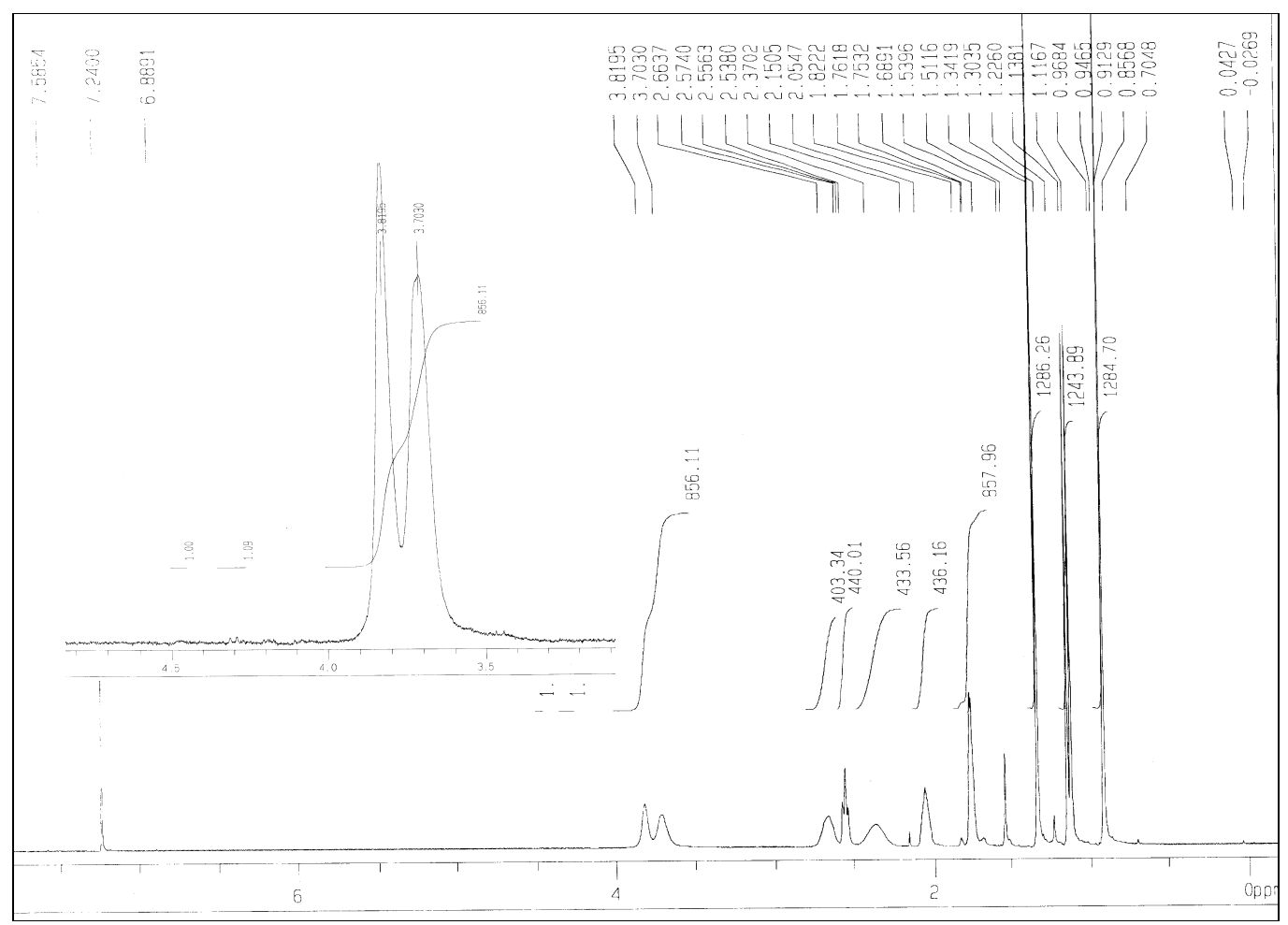

${ }^{13}$ C NMR 2a: $\left(\mathrm{CDCl}_{3}\right) \delta 136.0,72.2,71.0,40.5,39.9,37.8,33.8,29.9,26.1,21.7,13.9$.

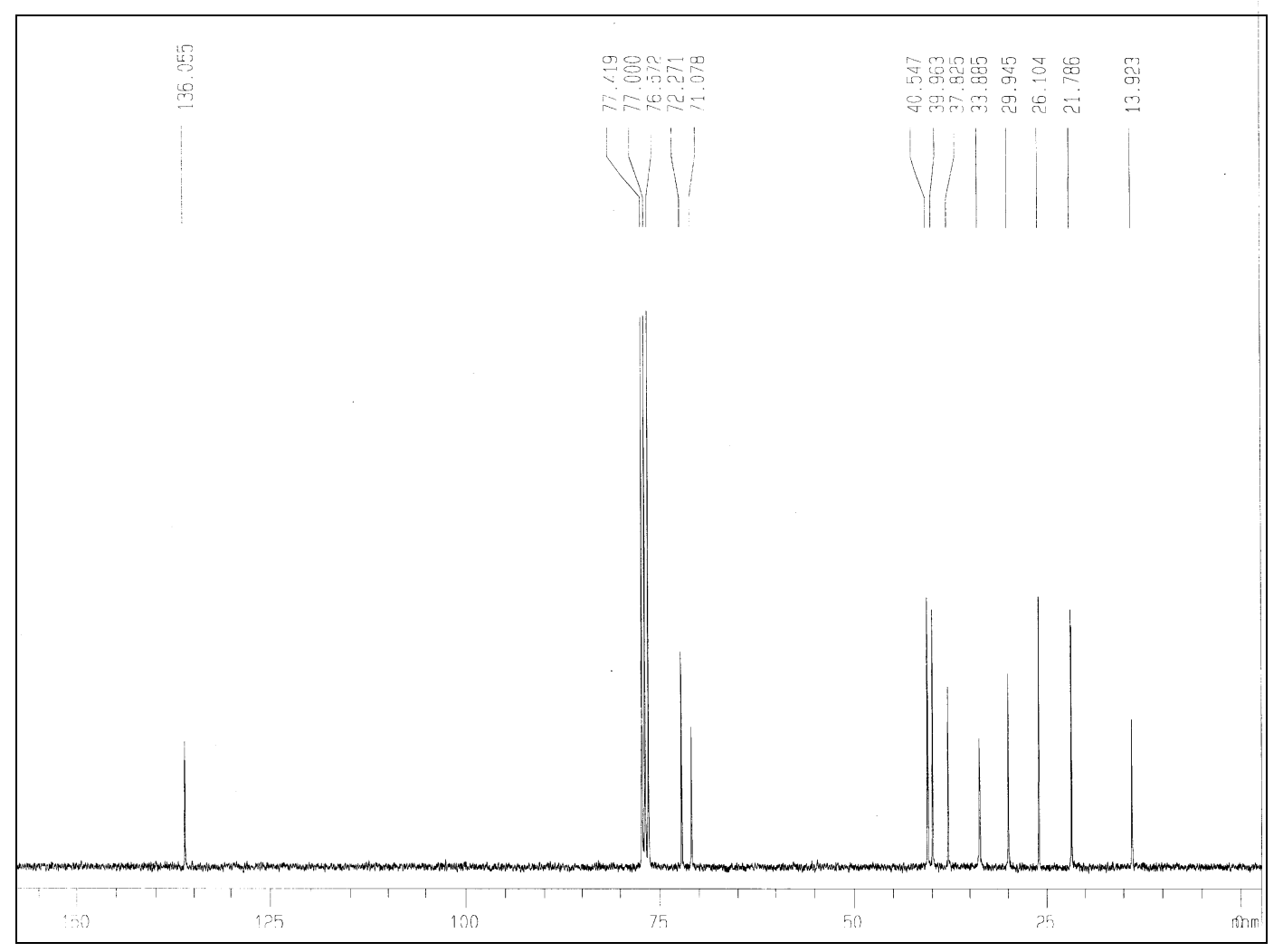


IR (KBr) 2982, 2951, 2912, 2870, 1499, 1466, 1448, 1429, 1367, 1265, 1219, 1101, 1051, 1032, 974, 860, 758 $\mathrm{cm}^{-1}$.

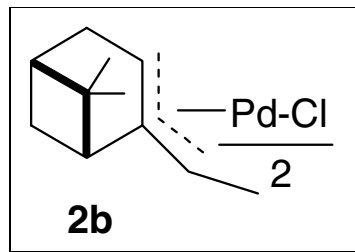

${ }^{1} \mathbf{H}$ NMR of $2 \mathbf{b}:\left(\mathrm{CDCl}_{3}\right) \delta 4.47-4.41(\mathrm{~m}, 1 \mathrm{H}), 4.33-4.22(\mathrm{~m}, 1 \mathrm{H}), 2.67-2.52(\mathrm{~m}, 1 \mathrm{H}), 2.36-2.3(\mathrm{~m}, 1 \mathrm{H})$, $2.2-2.13(\mathrm{~m}, 1 \mathrm{H}), 2.08-2.02(\mathrm{~m}, 1 \mathrm{H}), 1.87-1.74(\mathrm{~m}, 2 \mathrm{H}), 1.29(\mathrm{~s}, 3 \mathrm{H}), 1.03(\mathrm{~d}, J=6.8 \mathrm{~Hz}, 3 \mathrm{H}), 0.71(\mathrm{~s}, 3 \mathrm{H})$.

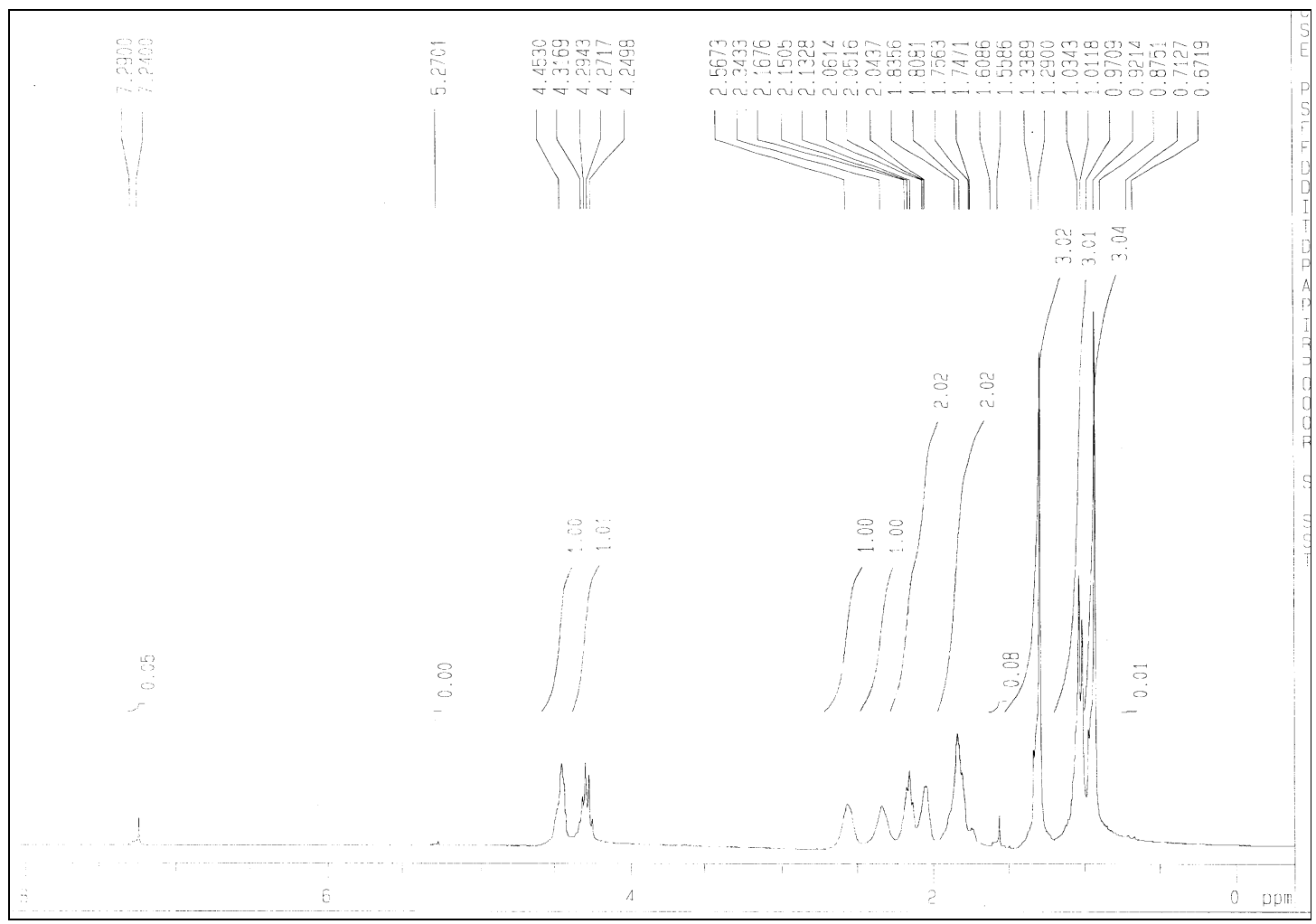


${ }^{13}$ C NMR 2b: $\left(\mathrm{CDCl}_{3}\right) \delta 132.5,72.7,70.8,46.5,39.9,38.0,34.4,29.9,26.0,21.7,16.2$.

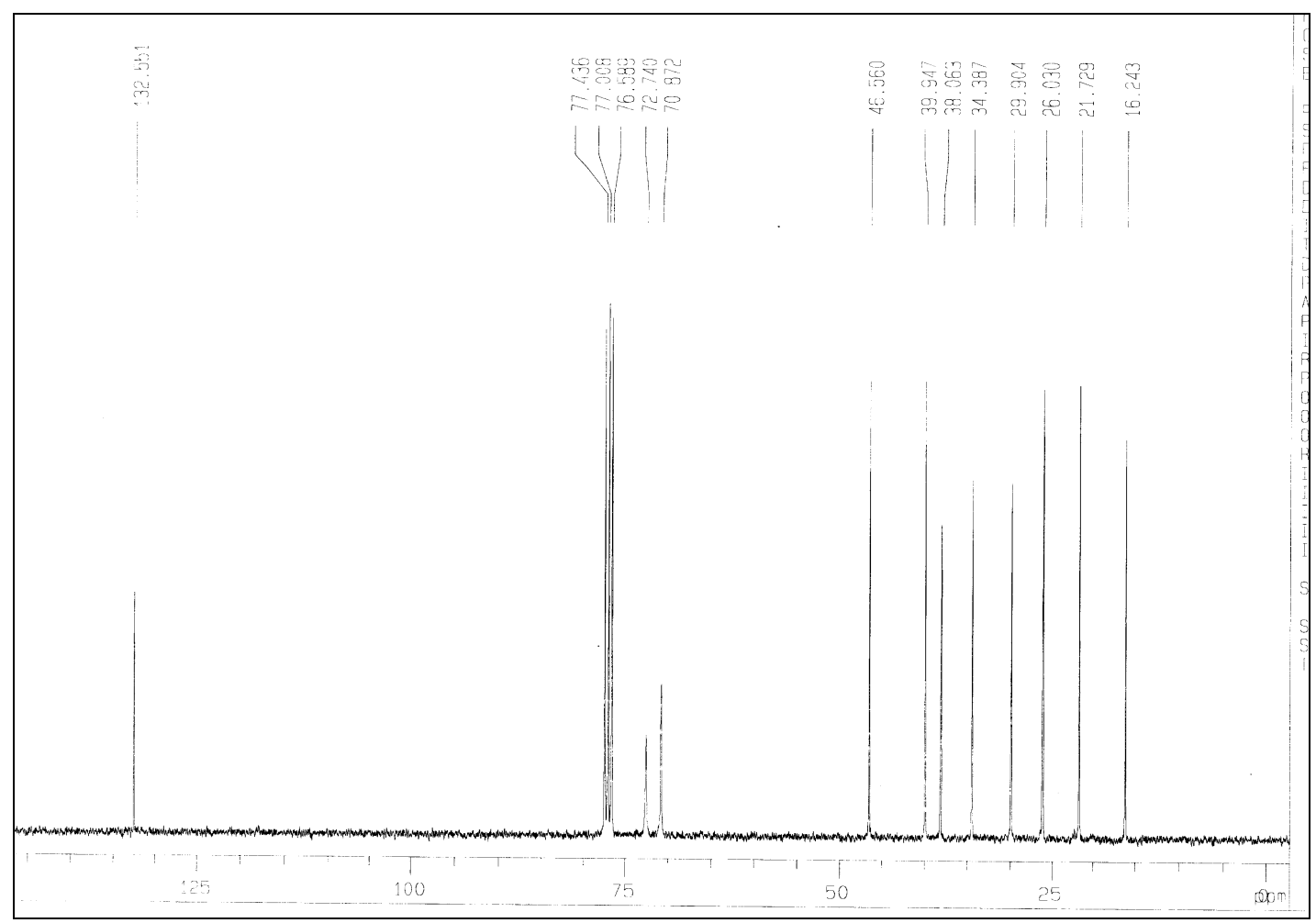

IR (KBr) 2980, 2918, 2872, 2833, 1468, 1454, 1429, 1369, 1265, 1221, 1099, 1042, 968, 920, $762 \mathrm{~cm}^{-1}$.

General equation for allylation of imines with allyltributylstannane and catalyst $2 a$

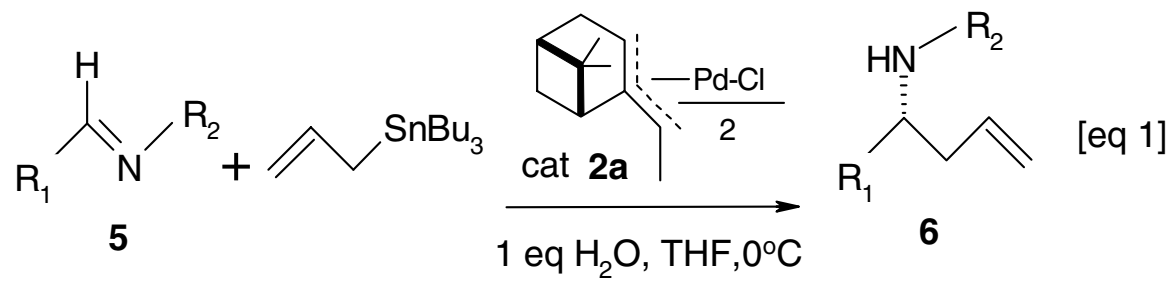


Table. Catalytic asymmetric allylation of imines with allyltribuylstannane in the presence of the catalyst $2 \mathbf{a}^{\mathrm{a}}$

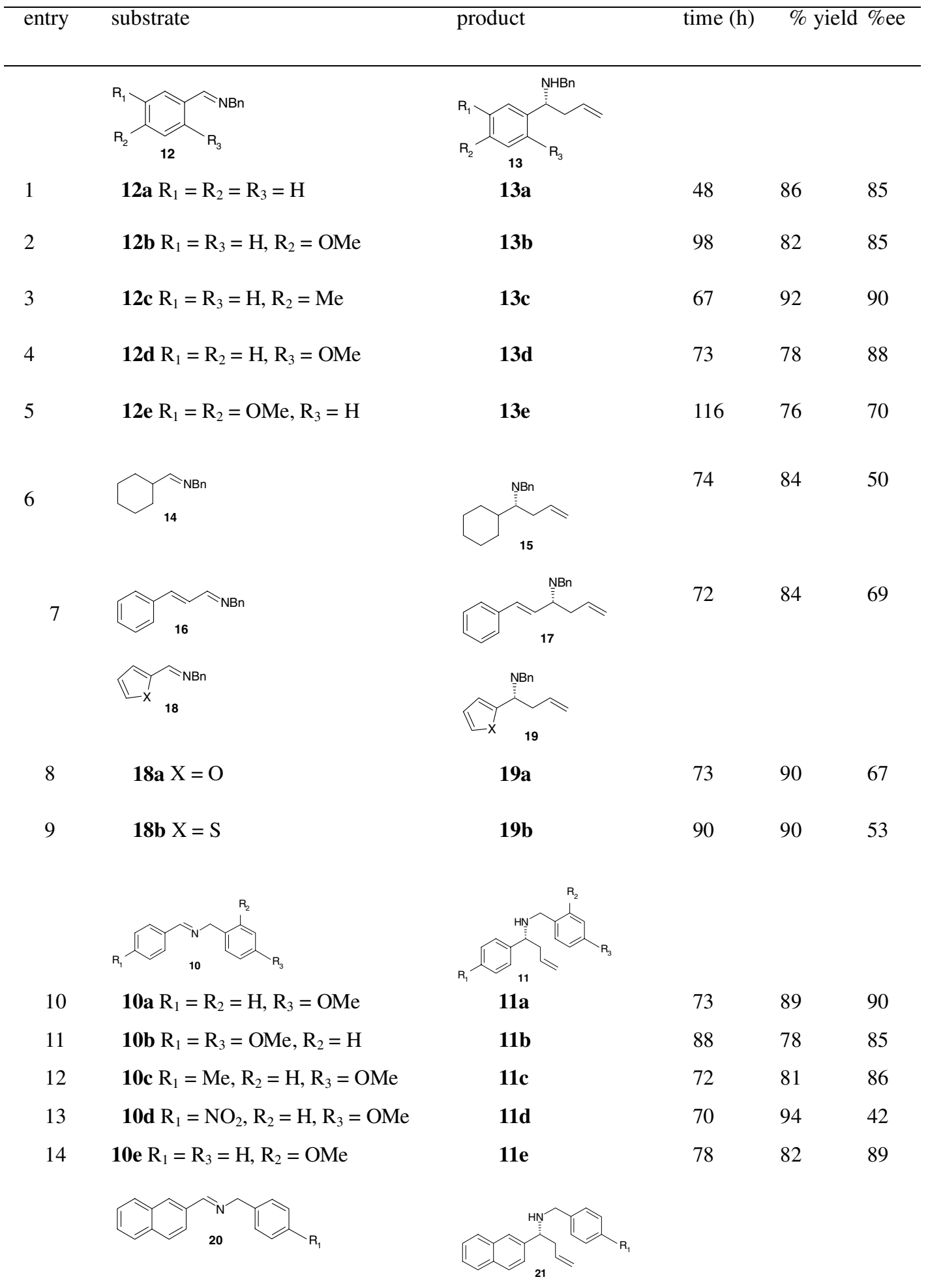




\begin{tabular}{|c|c|c|c|c|c|}
\hline 15 & $\mathbf{2 0 a} \mathrm{R}_{1}=\mathrm{H}$ & 21a & 96 & 70 & 91 \\
\hline 16 & 20b $\mathrm{R}_{1}=\mathrm{OMe}$ & 21b & 74 & 81 & 82 \\
\hline 17 & 22a R = allyl & $\mathbf{2 3 a}$ & 78 & 82 & 78 \\
\hline 18 & 22b $\mathrm{R}=\mathrm{SO}_{2} \mathrm{Ph}$ & 23b & 144 & $<10^{\mathrm{b}}$ & --- \\
\hline 19 & & & 168 & 42 & 55 \\
\hline 20 & & & 120 & 75 & 34 \\
\hline 21 & 8a $X=R_{1}=R_{2}=R_{3}=H$ & 9a & 84 & 76 & 90 \\
\hline 22 & $\mathbf{8 b} X=\mathrm{R}_{1}=\mathrm{R}_{2}=\mathrm{H}, \mathrm{R}_{3}=\mathrm{OMe}$ & $9 b$ & 72 & 76 & 89 \\
\hline 23 & 8c $X=R_{1}=R_{3}=H, R_{2}=O M e$ & $9 \mathrm{c}$ & 79 & 68 & 89 \\
\hline 24 & $\mathbf{8 d} X=\mathrm{Cl}, \mathrm{R}_{1}=\mathrm{R}_{2}=\mathrm{R}_{3}=\mathrm{H}$ & 9d & 89 & 84 & 87 \\
\hline 25 & $\mathbf{8 e} \mathrm{X}=\mathrm{Br}, \mathrm{R}_{1}=\mathrm{R}_{2}=\mathrm{R}_{3}=\mathrm{H}$ & $9 e$ & 99 & 87 & 88 \\
\hline 26 & 8f $X=R_{2}=R_{3}=H, R_{1}=P h$ & 9f & 126 & 30 & 83 \\
\hline \multirow[t]{2}{*}{27} & & & 20 & 38 & 2 \\
\hline & & & 20 & 63 & $18^{\mathrm{c}}$ \\
\hline \multirow[t]{2}{*}{28} & & & 41 & 77 & $7^{\mathrm{d}}$ \\
\hline & & & 43 & 73 & $19^{\mathrm{c}, \mathrm{d}}$ \\
\hline
\end{tabular}

${ }^{a}$ All reactions were carried out with 1.25 equivalent of allyltributylstannane and one equivalent of water in the presence of $5 \mathrm{~mol} \%$ of $\mathbf{2 a}$ and at $0^{\circ} \mathrm{C}$ for the specified time. In some cases additional 0.5 equivalent of allyltributylstannane was added if it was completely consumed and the reaction was not complete (checked by GC-MS); ${ }^{b}$ determined by ${ }^{1} \mathrm{H}$ NMR of the crude reaction mixture with $\mathrm{CH}_{3} \mathrm{NO}_{2}$ as internal standard. "using catalyst $\mathbf{2 b}$. ${ }^{\mathrm{d}}$ ee based on known $[\alpha]_{\mathrm{D}}$ values (see reference 6) 


\section{N-Benzyl-1-phenyl-3-butenylamine, 13a}

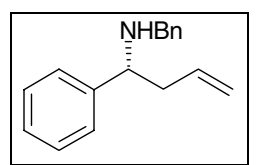

Colorless oil; $[\alpha]_{\mathrm{D}}{ }^{23}=+51.2\left(\mathrm{c} 1.45, \mathrm{CHCl}_{3}\right) ; \mathrm{IR},{ }^{1} \mathrm{H}$ NMR, ${ }^{13} \mathrm{C}$ NMR, HRMS and analysis data (see ref. 2). The enantiomeric excess was determined by using CHIRALCEL OD-R column, $\mathrm{CH}_{3} \mathrm{CN} / 1 \mathrm{M} \mathrm{NaClO}$ aq $=40 / 60$, flow rate $=0.8 \mathrm{~mL} / \mathrm{min}$, $\mathrm{UV}$ detection at $254 \mathrm{~nm}, \mathrm{t}_{\mathrm{R}}=16.38 \mathrm{~min}$ (major enantiomer), $\mathrm{t}_{\mathrm{R}}=18.48$ min (minor enantiomer); $85 \%$ ee.

\section{N-Benzyl-1-(4-methoxyphenyl)-3-butenylamine, 13b}

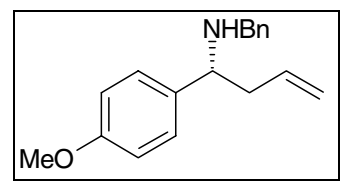

Colorless oil; $[\alpha]_{\mathrm{D}}{ }^{22}=+56.1\left(\mathrm{c} 0.745, \mathrm{CHCl}_{3}\right)$; IR, ${ }^{1} \mathrm{H}$ NMR,,${ }^{13} \mathrm{C}$ NMR, HRMS and analysis data (see ref. 2). The enantiomeric excess was determined by using CHIRALCEL OD-R column, $\mathrm{CH}_{3} \mathrm{CN} / 1 \mathrm{M} \mathrm{NaClO} \mathrm{aq}=40 / 60$, flow rate $=0.8 \mathrm{~mL} / \mathrm{min}$, $\mathrm{UV}$ detection at $254 \mathrm{~nm}, \mathrm{t}_{\mathrm{R}}=18.02 \mathrm{~min}$ (major enantiomer), $\mathrm{t}_{\mathrm{R}}=21.5 \mathrm{~min}$ (minor enantiomer); $85 \%$ ee.

\section{N-Benzyl-1-(4-methylphenyl)-3-butenylamine, 13c}

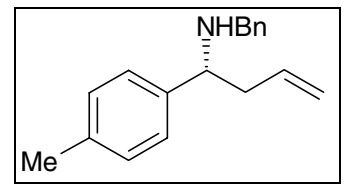

Colorless oil; $[\alpha]_{\mathrm{D}}^{22}=+50.4\left(\mathrm{c} 1.0, \mathrm{CHCl}_{3}\right.$ ); IR (neat) 3325, 3061, 3025, 2977, 2921, 1638, 1603, 1513, 1495, 1454, 1350, 1304, 1176, 1029, 995, 916, 816, $735 \mathrm{~cm}^{-1} ;{ }^{1} \mathrm{H}$ NMR $\left(\mathrm{CDCl}_{3}\right) \delta 7.28-7.13(\mathrm{~m}, 7 \mathrm{H}), 7.09$ (d, $J=8 \mathrm{~Hz}$, 2H), 5.6 (ddt, $J=17.1 \mathrm{~Hz}, 10.3 \mathrm{~Hz}, 7.1 \mathrm{~Hz}, 1 \mathrm{H}$ ), 5.02 (ddt, $J=17.1 \mathrm{~Hz}, 2 \mathrm{~Hz}, 2 \mathrm{~Hz}, 1 \mathrm{H}$ ), 4.97 (ddt, $J=10.3 \mathrm{~Hz}$, $2 \mathrm{~Hz}, 2 \mathrm{~Hz}, 1 \mathrm{H}), 3.61-3.59$ (m, 1H), $3.6(\mathrm{~d}, J=13.4 \mathrm{~Hz}, 1 \mathrm{H}), 3.45$ (d, $J=13.4 \mathrm{~Hz}, 1 \mathrm{H}), 2.34-2.32$ (m, 2H), 2.3 (s, 3H), 1.66 (brs, 1H); 


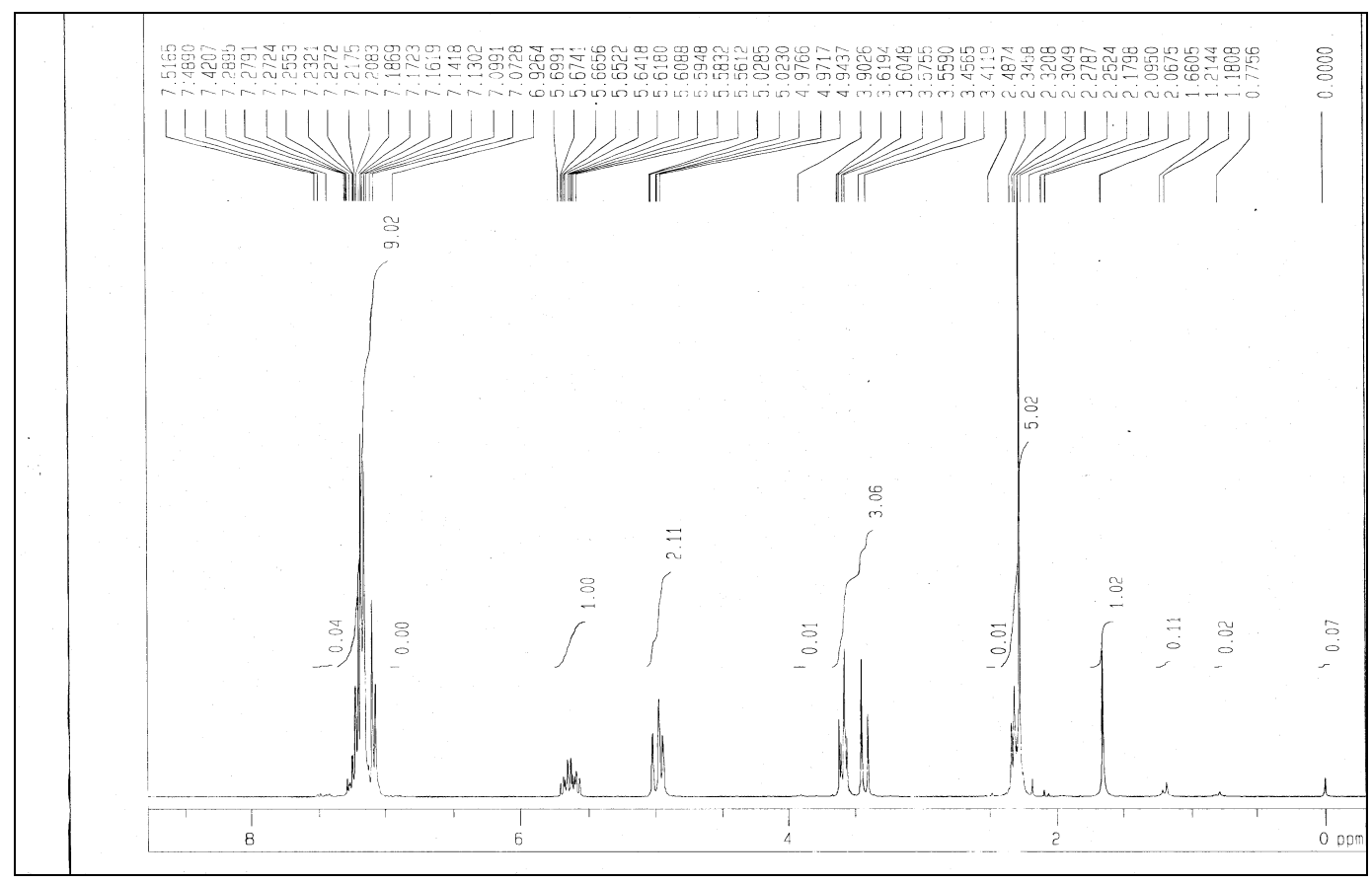

${ }^{13} \mathrm{C} \mathrm{NMR}\left(\mathrm{CDCl}_{3}\right) \delta 140.7,136.5,135.5,129.0,128.2,128.0,127.2,126.7,117.4,61.2,51.3,43.1,21.0$; MS (EI) $\mathrm{m} / z$ (relative intensity) 210 [M+-allyl] (5.7), 132 (0.7), 118 (1.2), 105 (2.9), 91 (100); Anal. Calcd for $\mathrm{C}_{18} \mathrm{H}_{21} \mathrm{~N}$ (251.37): $\mathrm{C}, 86.00 ; \mathrm{H}, 8.42$; N, 5.57. Found: $\mathrm{C}, 85.82 ; \mathrm{H}, 8.66$; N, 5.52. The enantiomeric excess was determined using CHIRALCEL OD-R column, $\mathrm{CH}_{3} \mathrm{CN} / 1 \mathrm{M} \mathrm{NaClO}{ }_{4}$ aq $=40 / 60$, flow rate $=0.8 \mathrm{~mL} / \mathrm{min}$, UV detection at $254 \mathrm{~nm}, \mathrm{t}_{\mathrm{R}}=22.82 \mathrm{~min}$ (major enantiomer), $\mathrm{t}_{\mathrm{R}}=27.20 \mathrm{~min}$ (minor enantiomer); $90 \%$ ee.

\section{N-Benzyl-1-(2-methoxyphenyl)-3-butenylamine, 13d}

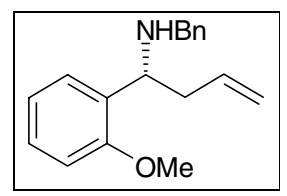

Colorless oil; $[\alpha]_{\mathrm{D}}^{22}=+37.2\left(\mathrm{c} 1.0, \mathrm{CHCl}_{3}\right)$; IR (neat) 3330, 3063, 3027, 3001, 2935, 2835, 1638, 1600, 1586, 1489, 1437, 1363, 1281, 1239, 1173, 1095, 1050, 1029, 996, 914, $754 \mathrm{~cm}^{-1} ;{ }^{1} \mathrm{H}$ NMR $\left(\mathrm{CDCl}_{3}\right) \delta$ 7.38-7.35 (m, $1 \mathrm{H}), 7.26-7.16(\mathrm{~m}, 6 \mathrm{H}), 6.95-6.83(\mathrm{~m}, 2 \mathrm{H}), 5.72(\mathrm{ddt}, J=16.1 \mathrm{~Hz}, 10.2 \mathrm{~Hz}, 6.7 \mathrm{~Hz}, 1 \mathrm{H}), 5.03-4.93(\mathrm{~m}, 2 \mathrm{H})$, 4.07-4.03 (m, 1H), $3.77(\mathrm{~s}, 3 \mathrm{H}), 3.65(\mathrm{~d}, J=13 \mathrm{~Hz}, 1 \mathrm{H}), 3.51(\mathrm{~d}, J=13 \mathrm{~Hz}, 1 \mathrm{H}), 2.5-2.34(\mathrm{~m}, 2 \mathrm{H}), 1.8$ (brs, $1 \mathrm{H})$ 


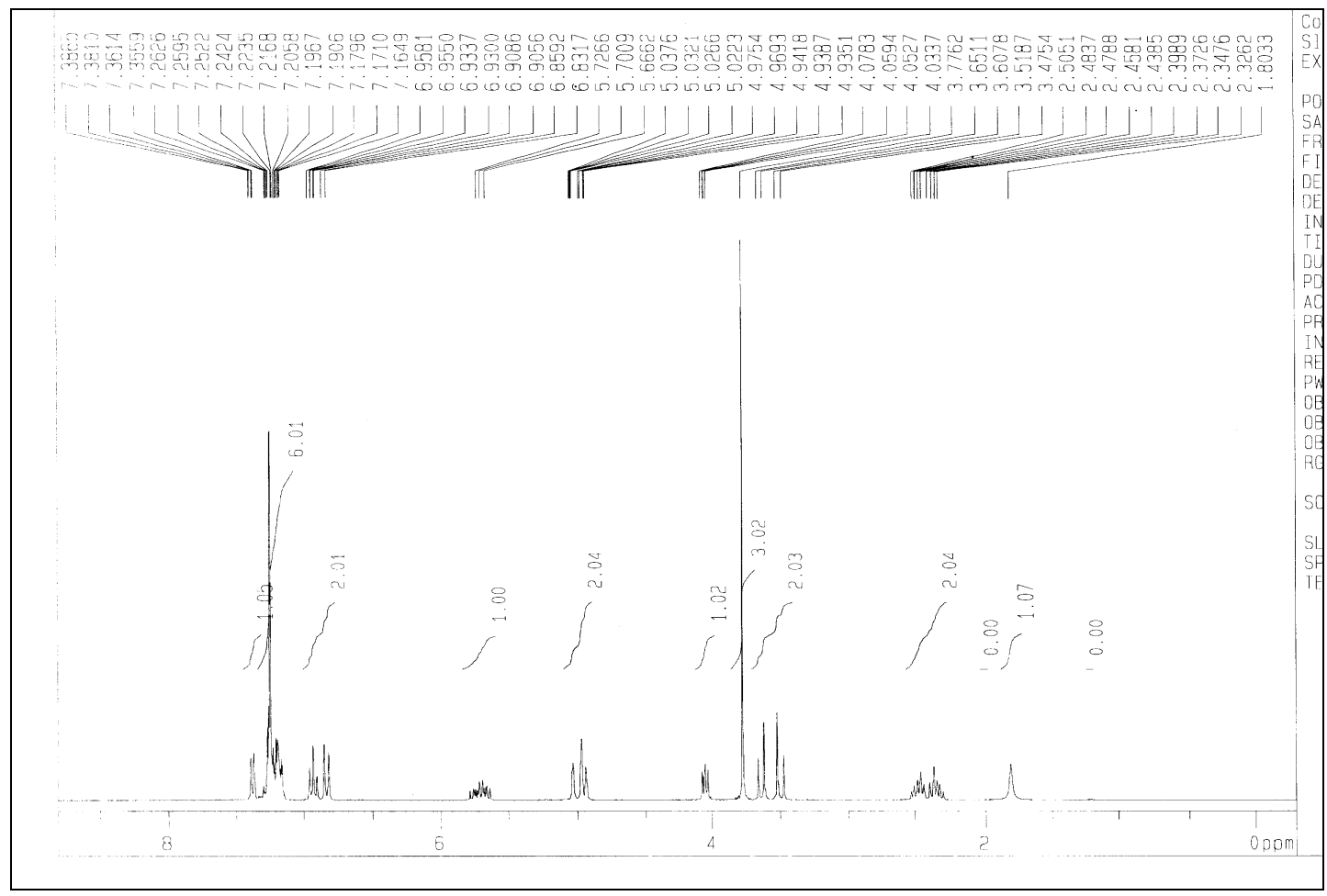

${ }^{13} \mathrm{C} \mathrm{NMR}\left(\mathrm{CDCl}_{3}\right) \delta 157.2,140.7,136.0,131.2,128.0,127.9,127.7,127.4,127.1,126.5,120.4,116.6,110.3$, 55.8, 55.0, 51.4, 40.7; MS (EI) m/z (relative intensity) 226 [M+allyl] (15.8), 209 (6.7), 197 (3.1), 194 (4.9), 134 (3.8), 121 (17.6), 91 (100); Anal. Calcd for $\mathrm{C}_{18} \mathrm{H}_{21} \mathrm{NO}$ (267.37): C, 80.86; H, 7.91; N, 5.23. Found: C, 80.81; H, $8.11 ; \mathrm{N}, 5.28$. The enantiomeric excess was determined by converting to trifluoroacetylamide form, $[\alpha]_{\mathrm{D}}{ }^{22}=+$ 31.0 (c 2.8, $\mathrm{CHCl}_{3}$ ) and using CHIRALCEL OD column, hexane $/ i$-PrOH $=100 / 1$, flow rate $=0.7 \mathrm{~mL} / \mathrm{min}$, UV detection at $254 \mathrm{~nm}, \mathrm{t}_{\mathrm{R}}=9.04 \mathrm{~min}$ (major enantiomer), $\mathrm{t}_{\mathrm{R}}=10.43 \mathrm{~min}$ (minor enantiomer); $88 \%$ ee.

\section{N-Benzyl-1-(3,4-dimethoxyphenyl)-3-butenylamine, 13e}

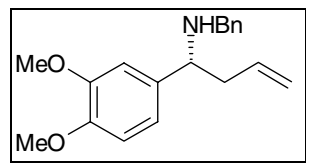

Colorless oil; $[\alpha]_{\mathrm{D}}{ }^{23}=+38.6$ (c 1.75, $\mathrm{CHCl}_{3}$ ); IR (neat) 3325, 3062, 2998, 2932, 2833, 1638, 1593, 1514, 1463, $1417,1358,1262,1233,1139,1029,916,856,808,747 \mathrm{~cm}^{-1} ;{ }^{1} \mathrm{H}^{\mathrm{NMR}}\left(\mathrm{CDCl}_{3}\right) \delta 7.32-7.19(\mathrm{~m}, 5 \mathrm{H}), 6.95-6.81$ (m, 3H), 5.72 (ddt, $J=16.2 \mathrm{~Hz}, 10.2 \mathrm{~Hz}, 7.2 \mathrm{~Hz}, 1 \mathrm{H}), 5.06$ (ddt, $J=16.2 \mathrm{~Hz}, 2 \mathrm{~Hz}, 2 \mathrm{~Hz}, 1 \mathrm{H}), 5.03$ (ddt, $J=10.2$ $\mathrm{Hz}, 2 \mathrm{~Hz}, 2 \mathrm{~Hz}, 1 \mathrm{H}), 3.9$ (s, 3H), 3.88 (s, 3H), 3.69 (d, $J=13.2 \mathrm{~Hz}, 1 \mathrm{H}), 3.63-3.59$ (m, 1H), 3.54 (d, $J=13.2 \mathrm{~Hz}$, $1 \mathrm{H}), 2.41-2.34(\mathrm{~m}, 2 \mathrm{H}), 1.81$ (brs, $1 \mathrm{H})$; 


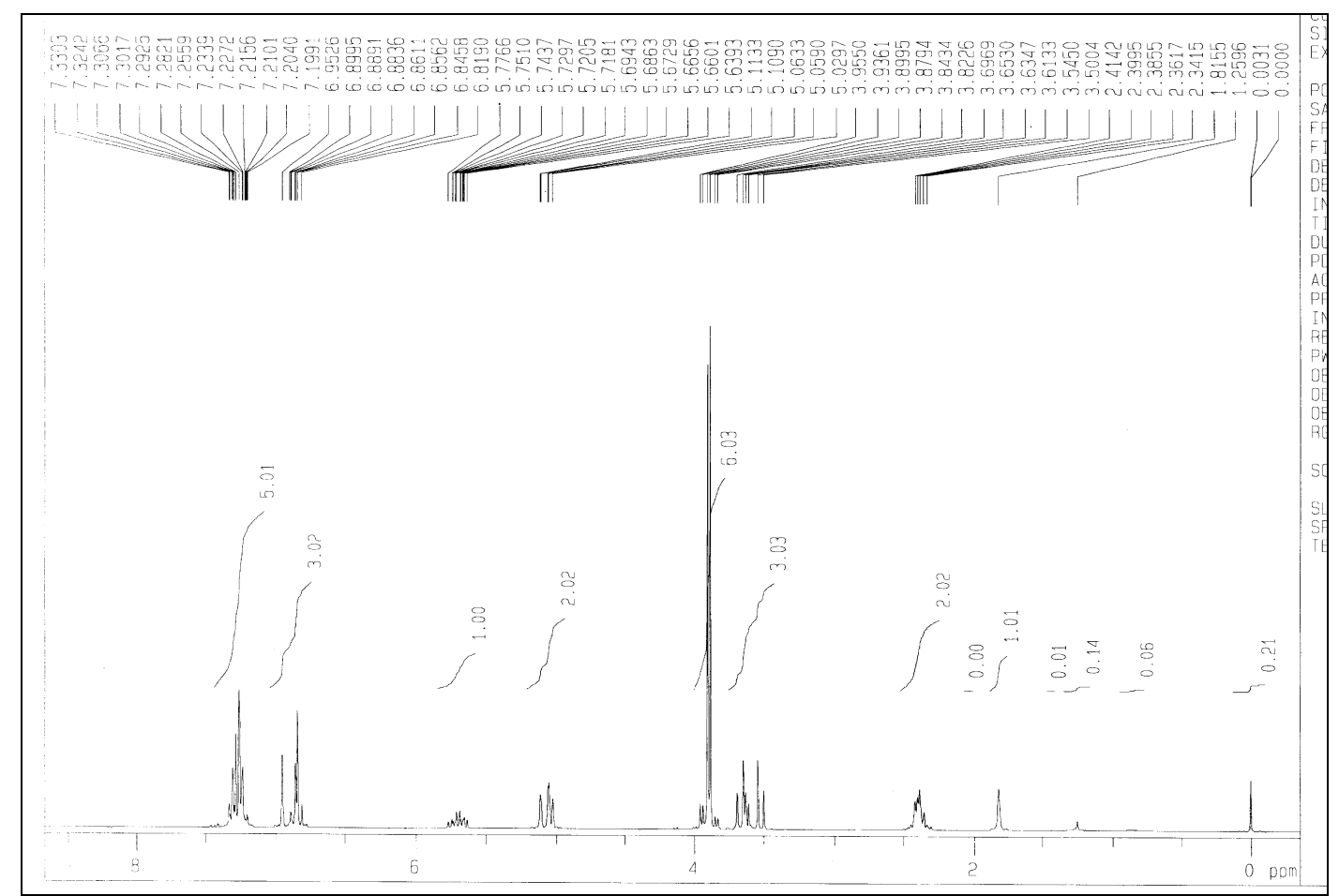

${ }^{13} \mathrm{C} \mathrm{NMR}\left(\mathrm{CDCl}_{3}\right) \delta 149.0,147.9,140.6,136.3,135.5,128.3,128.1,126.8,119.5,117.5,110.8,109.9,61.2,55.8$, 51.3, 43.2; MS (EI) m/z (relative intensity) 256 [ $\mathrm{M}^{+}$-allyl] (14.8), 239 (5), 214 (9.4), 151 (20.8), 91 (100); Anal. Calcd for $\mathrm{C}_{19} \mathrm{H}_{23} \mathrm{NO}_{2}$ (297.39): C, 76.73; H, 7.79; N, 4.71. Found: C, 76.68; H, 8.06; N, 4.72. The enantiomeric excess was determined by using CHIRALCEL OD-R column, $\mathrm{CH}_{3} \mathrm{CN} / 1 \mathrm{M} \mathrm{NaClO}{ }_{4}$ aq $=30 / 70$, flow rate $=0.8$ $\mathrm{mL} / \mathrm{min}$, UV detection at $254 \mathrm{~nm}, \mathrm{t}_{\mathrm{R}}=28.57 \mathrm{~min}$ (major enantiomer), $\mathrm{t}_{\mathrm{R}}=32.58 \mathrm{~min}$ (minor enantiomer); $70 \%$ ee.

\section{N-Benzyl-1-cyclohexyl-3-butenylamine, 15}

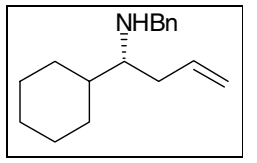

Colorless oil; $[\alpha]_{\mathrm{D}}{ }^{21}=-5.4\left(\mathrm{c} 0.5, \mathrm{CHCl}_{3}\right)$; IR, ${ }^{1} \mathrm{H}$ NMR, ${ }^{13} \mathrm{C}$ NMR, HRMS and analysis data (see ref. 2). The enantiomeric excess was determined by converting to trifluoroacetylamide form, $[\alpha]_{\mathrm{D}}^{22}=-7.8$ (c $0.6, \mathrm{CHCl}_{3}$ ) and using CHIRALCEL OD column, hexane $/ i-\mathrm{PrOH}=800 / 1$, flow rate $=0.6 \mathrm{~mL} / \mathrm{min}$, UV detection at $254 \mathrm{~nm}$, $t_{R}=26.04 \min$ (minor enantiomer), $t_{R}=30.41$ min (major enantiomer); $50 \%$ ee.

\section{N-Benzyl-1-phenyl-1,5-hexadien-3-ylamine, 17}

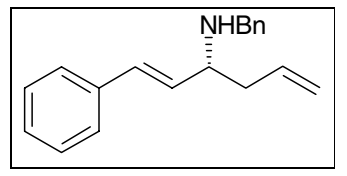

Colorless oil; $[\alpha]_{\mathrm{D}}{ }^{23}=+77.2\left(\mathrm{c} 1.0, \mathrm{CHCl}_{3}\right)$; IR, ${ }^{1} \mathrm{H}$ NMR, ${ }^{13} \mathrm{C}$ NMR, HRMS and analysis data (see ref. 2). The 
enantiomeric excess was determined using CHIRALCEL OD-R column, $\mathrm{CH}_{3} \mathrm{CN} / 1 \mathrm{M} \mathrm{NaClO} 4 \mathrm{aq}=60 / 40$, flow rate $=0.6 \mathrm{~mL} / \mathrm{min}, \mathrm{UV}$ detection at $254 \mathrm{~nm}, \mathrm{t}_{\mathrm{R}}=10.03 \mathrm{~min}$ (major enantiomer), $\mathrm{t}_{\mathrm{R}}=12.17$ min (minor enantiomer); $69 \%$ ee.

\section{N-Benzyl-1-(2-furfuryl)-3-butenylamine, 19a}

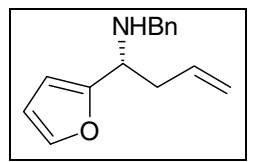

Colorless oil; $[\alpha]_{\mathrm{D}}^{22}=+55.9\left(\mathrm{c} 1.0, \mathrm{CHCl}_{3}\right)$; IR (neat) 3330, 3063, 3028, 2978, 2914, 2837, 1640, 1604, 1512, $1495,1455,1346,1248,1176,1148,1108,1073,1028,918,884,807,734 \mathrm{~cm}^{-1} ;{ }^{1} \mathrm{H}$ NMR $\left(\mathrm{CDCl}_{3}\right) \delta 7.31-7.13$ $(\mathrm{m}, 6 \mathrm{H}), 6.25(\mathrm{dd}, J=3.2 \mathrm{~Hz}, 2 \mathrm{~Hz}, 1 \mathrm{H}), 6.11(\mathrm{~d}, J=3.2 \mathrm{~Hz}, 1 \mathrm{H}), 5.68$ (ddt, $J=17.1 \mathrm{~Hz}, 10.2 \mathrm{~Hz}, 6.6 \mathrm{~Hz}, 1 \mathrm{H})$, 5.05 (ddt, $J=17.1 \mathrm{~Hz}, 2.2 \mathrm{~Hz}, 2 \mathrm{~Hz}, 1 \mathrm{H}), 5.03$ (ddt, $J=10.2 \mathrm{~Hz}, 2.2 \mathrm{~Hz}, 2 \mathrm{~Hz}, 1 \mathrm{H}), 3.72-3.70$ (m, $1 \mathrm{H}), 3.69$ (d, $J=13.2 \mathrm{~Hz}, 1 \mathrm{H}), 3.54(\mathrm{~d}, J=13.2 \mathrm{~Hz}, 1 \mathrm{H}), 2.46(\mathrm{dd}, J=6.6 \mathrm{~Hz}, 1.3 \mathrm{~Hz}, 2 \mathrm{H}), 1.65$ (brs, $1 \mathrm{H})$;

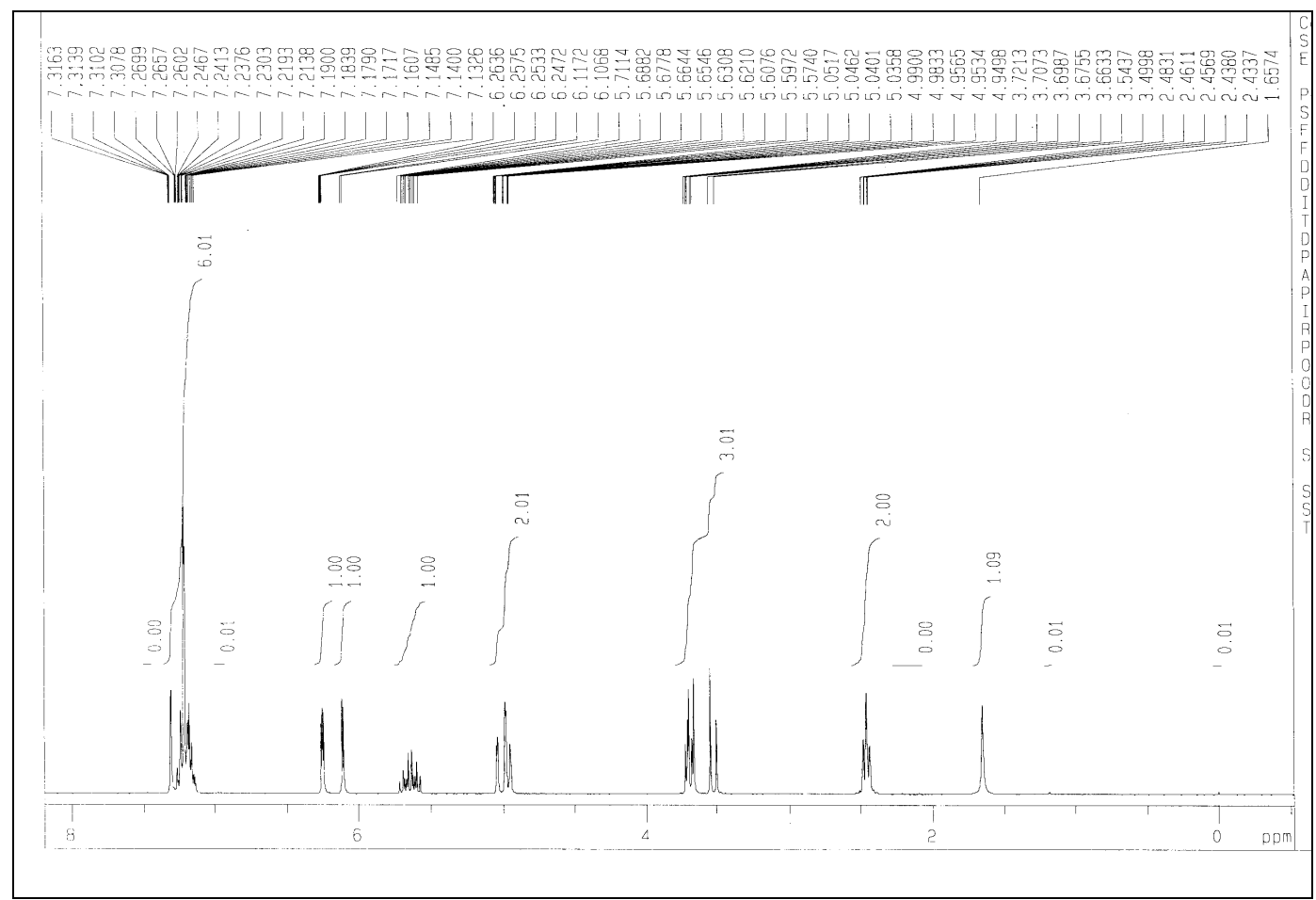

${ }^{13} \mathrm{C} \mathrm{NMR}\left(\mathrm{CDCl}_{3}\right) \delta 156.0,141.4,140.1,134.8,128.2,128.0,126.7,117.5,109.7,106.5,54.7,50.93,39.2 ; \mathrm{MS}$ (EI) $\mathrm{m} / z$ (relative intensity) $186\left[\mathrm{M}^{+}-\right.$allyl $]$(33.9), 131 (1.5), 95 (1.8), 91 (100); Anal. Calcd for $\mathrm{C}_{15} \mathrm{H}_{17} \mathrm{NO}$ (227.3): C, 79.26; H, 7.53; N, 6.16. Found: C, 79.11; H, 7.84; N, 6.15. The enantiomeric excess was determined by converting to trifluoroacetylamide form, $[\alpha]_{\mathrm{D}}{ }^{22}=+54.7\left(\mathrm{c} 1.0, \mathrm{CHCl}_{3}\right)$ and using CHIRALCEL OD column, hexane $/ i-\mathrm{PrOH}=500 / 1$, flow rate $=0.5 \mathrm{~mL} / \mathrm{min}, \mathrm{UV}$ detection at $254 \mathrm{~nm}, \mathrm{t}_{\mathrm{R}}=22.18$ min (major enantiomer), $\mathrm{t}_{\mathrm{R}}$ $=25.2 \mathrm{~min}$ (minor enantiomer); $67 \%$ ee. 


\section{N-Benzyl-1-(2-thiophenyl)-3-butenylamine, 19b}

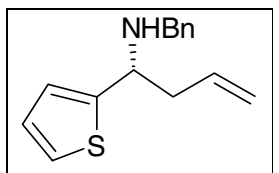

Colorless oil; $[\alpha]_{\mathrm{D}}^{22}=+24.5\left(\mathrm{c} 1.0, \mathrm{CHCl}_{3}\right.$ ); IR (neat) 3325, 3064, 3027, 2977, 2910, 2835, 1639, 1603, 1495, $1454,1434,1368,1225,1168,1029,995,917,850,735 \mathrm{~cm}^{-1} ;{ }^{1} \mathrm{H}$ NMR $\left(\mathrm{CDCl}_{3}\right) \delta 7.27-7.14(\mathrm{~m}, 6 \mathrm{H}), 6.9-6.84$ $(\mathrm{m}, 2 \mathrm{H}), 5.69$ (ddt, $J=16.1 \mathrm{~Hz}, 10.0 \mathrm{~Hz}, 6.9 \mathrm{~Hz}, 1 \mathrm{H}), 5.05$ (ddt, $J=16.1 \mathrm{~Hz}, 2.1 \mathrm{~Hz}, 2 \mathrm{~Hz}, 1 \mathrm{H}), 5.0(\mathrm{ddt}, J=$ $10.0 \mathrm{~Hz}, 2.1 \mathrm{~Hz}, 2 \mathrm{~Hz}, 1 \mathrm{H}), 3.92(\mathrm{t}, J=6.7 \mathrm{~Hz}, 1 \mathrm{H}), 3.74(\mathrm{~d}, J=13.2 \mathrm{~Hz}, 1 \mathrm{H}), 3.55(\mathrm{~d}, J=13.2 \mathrm{~Hz}, 1 \mathrm{H})$, $2.44-2.34(\mathrm{~m}, 2 \mathrm{H}), 1.73$ (brs, $1 \mathrm{H})$;

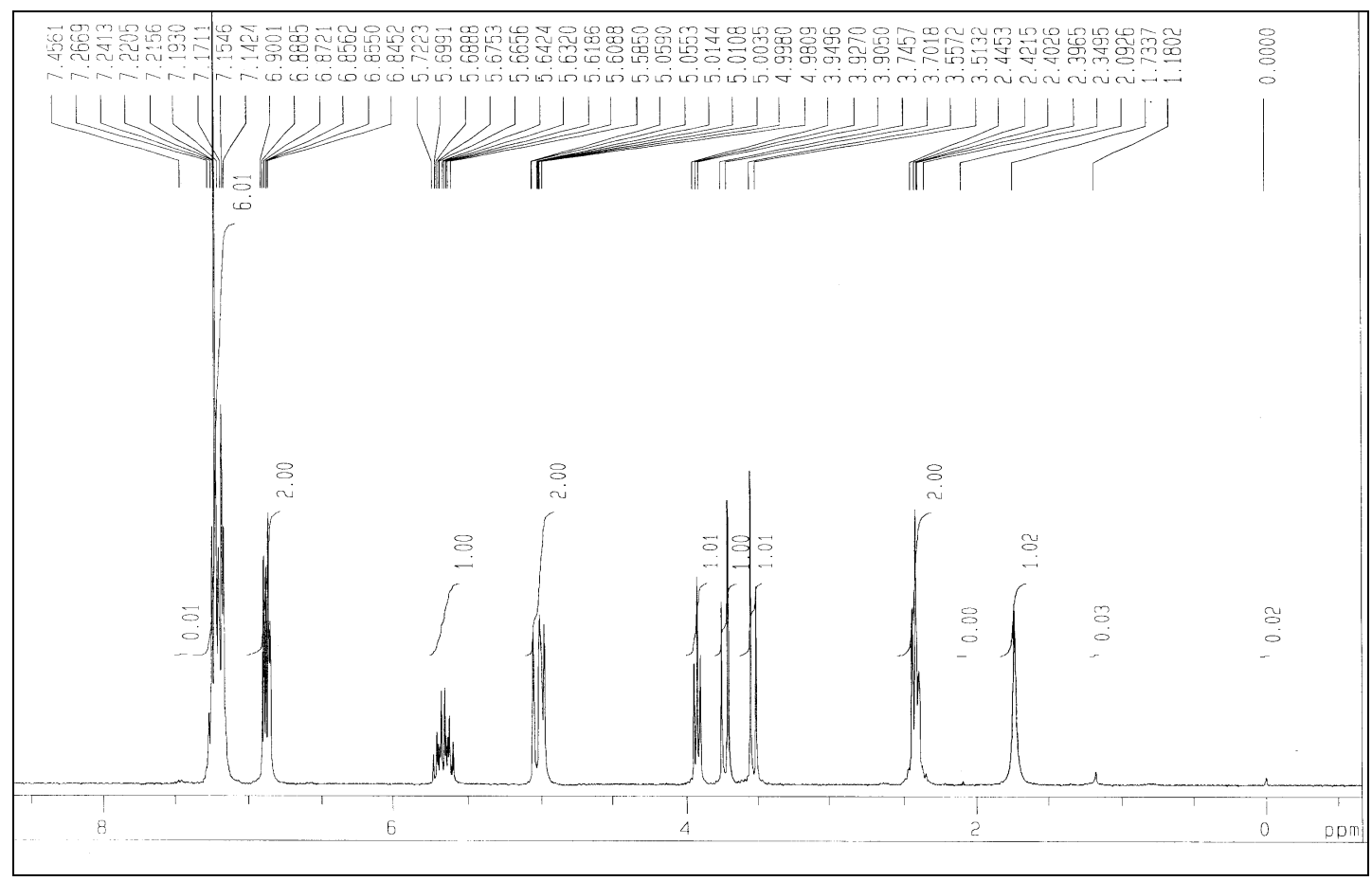

${ }^{13} \mathrm{C} \mathrm{NMR}\left(\mathrm{CDCl}_{3}\right) \delta 149.2,140.2,134.8,128.3,128.2,126.9,126.3,124.2,123.9,117.9,57.0 ; \mathrm{MS}(\mathrm{EI}) \mathrm{m} / z$ (relative intensity) 202 [M $\mathrm{M}^{+}$-allyl] (18.9), 111 (2), 106 (1.3), 97 (7.9), 91 (100); Anal. Calcd for $\mathrm{C}_{15} \mathrm{H}_{17} \mathrm{NS}$ (243.37): C, 74.03; H, 7.04; N, 5.75; S, 13.17. Found: C, 73.96; H, 7.32; N, 5.66; S, 13.18. The enantiomeric excess was determined by converting to trifluoroacetylamide form, $[\alpha]_{\mathrm{D}}{ }^{22}=+43.4\left(\mathrm{c} 1.0, \mathrm{CHCl}_{3}\right)$ and using CHIRALCEL OD column, hexane $/ i-\mathrm{PrOH}=500 / 1$, flow rate $=0.5 \mathrm{~mL} / \mathrm{min}, \mathrm{UV}$ detection at $254 \mathrm{~nm}, \mathrm{t}_{\mathrm{R}}=27.55$ $\min$ (major enantiomer), $\mathrm{t}_{\mathrm{R}}=30.59 \mathrm{~min}$ (minor enantiomer); $53 \%$ ee.

\section{N-(4-Methoxybenzyl)-1-phenyl-3-butenylamine, 11a}

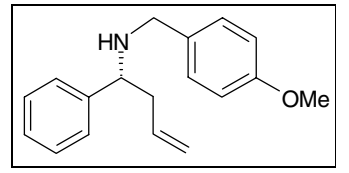


Colorless oil; $[\alpha]_{\mathrm{D}}{ }^{23}=+49.1\left(\mathrm{c} 1.0, \mathrm{CHCl}_{3}\right)$; IR, ${ }^{1} \mathrm{H}$ NMR, ${ }^{13} \mathrm{C}$ NMR, HRMS and analysis data (see ref. 2). The enantiomeric excess was determined by converting to trifluoroacetylamide form, $[\alpha]_{\mathrm{D}}{ }^{22}=+75.0\left(\mathrm{c} 1.75, \mathrm{CHCl}_{3}\right)$ and using CHIRALCEL OD column, hexane $/ i-\mathrm{PrOH}=100 / 1$, flow rate $=0.6 \mathrm{~mL} / \mathrm{min}$, UV detection at $254 \mathrm{~nm}$, $t_{R}=17.02 \min$ (major enantiomer), $t_{R}=20.19 \min$ (minor enantiomer); $90 \%$ ee.

N-(4-Methoxybenzyl)-1-(4-methoxyphenyl)-3-butenylamine, 11b

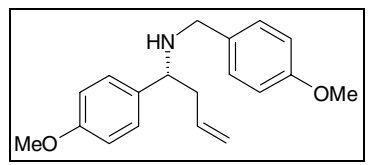

Colorless oil; $[\alpha]_{\mathrm{D}}^{22}=+48.3\left(\mathrm{c} 1.0, \mathrm{CHCl}_{3}\right.$ ); IR (neat) 3325, 3072, 2998, 2932, 2907, 2834, 2059, 1638, 1611, $1585,1512,1463,1351,1301,1245,1174,1106,1036,997,917,831,781,756 \mathrm{~cm}^{-1} ;{ }^{1} \mathrm{H} \mathrm{NMR}\left(\mathrm{CDCl}_{3}\right) \delta 7.26$ $(\mathrm{d}, J=8.6 \mathrm{~Hz}, 2 \mathrm{H}), 7.15(\mathrm{~d}, J=8.6 \mathrm{~Hz}, 2 \mathrm{H}), 6.89(\mathrm{~d}, J=8.6 \mathrm{~Hz}, 2 \mathrm{H}), 6.84(\mathrm{~d}, J=8.6 \mathrm{~Hz}, 2 \mathrm{H}), 5.7$ (ddt, $J=16.2$ $\mathrm{Hz}, 10.1 \mathrm{~Hz}, 6.5 \mathrm{~Hz}, 1 \mathrm{H}), 5.07-4.9$ (m, 2H), 3.8 (s, 3H), 3.78 (s, 3H), 3.63-3.61 (m, 1H), 3.58 (d, J=13 Hz, 1H), $3.44(\mathrm{~d}, J=13 \mathrm{~Hz}, 1 \mathrm{H}), 2.38-2.33(\mathrm{~m}, 2 \mathrm{H}), 1.69$ (brs, 1H);

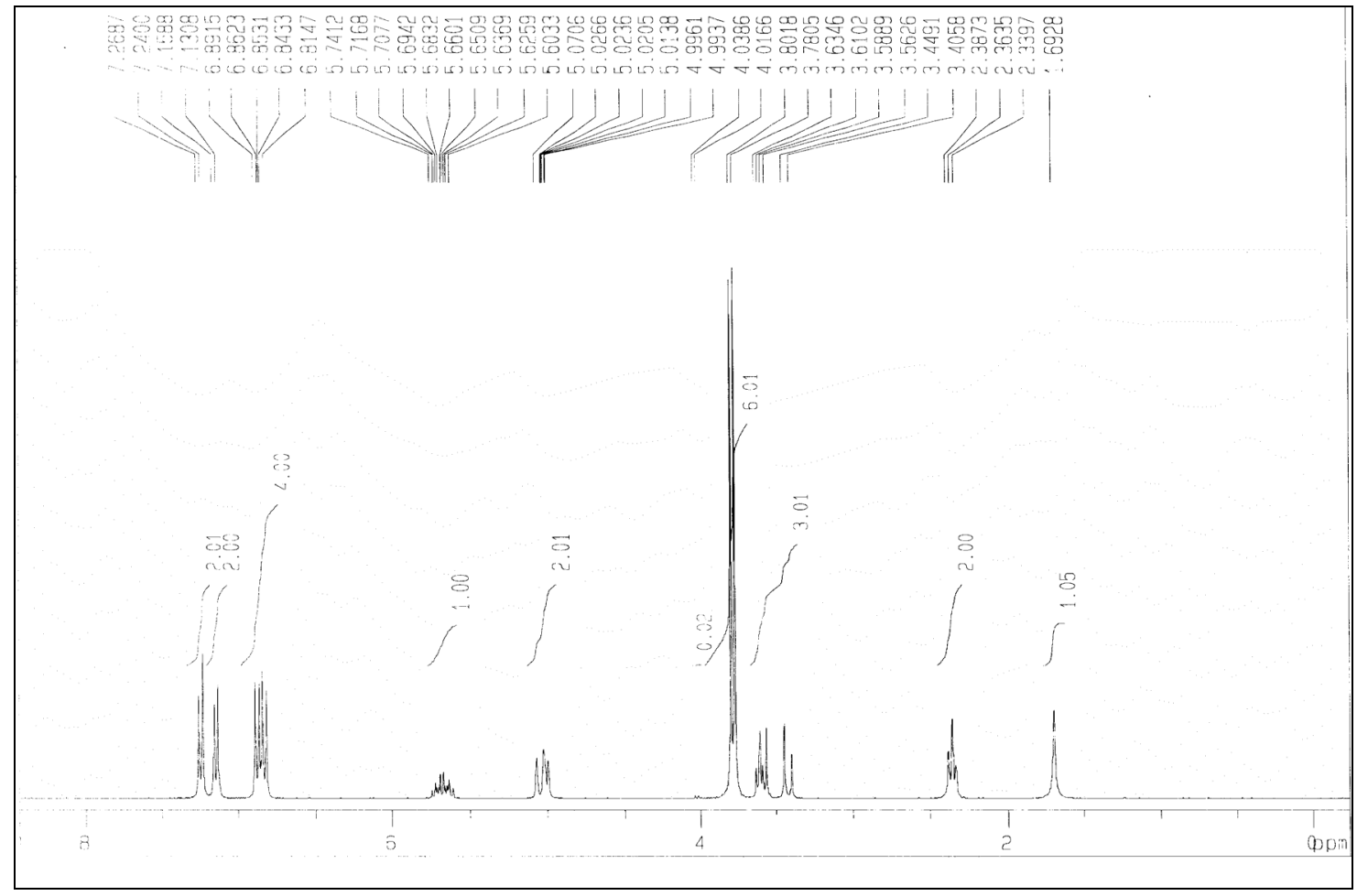

${ }^{13} \mathrm{C} \mathrm{NMR}\left(\mathrm{CDCl}_{3}\right) \delta 158.5,135.7,135.6,132.7,129.2,128.2,117.3,113.6,60.7,55.2,55.1,50.6,43.1 ; \mathrm{MS}$ (EI) $\mathrm{m} / z$ (relative intensity) 256 [M+-allyl] (9.4), 160 (6), 122 (8.8), 121 (100), 91 (6.7), 77 (2.9); Anal. Calcd for $\mathrm{C}_{19} \mathrm{H}_{23} \mathrm{NO}_{2}$ (297.39): C, 76.73; H, 7.79; N, 4.71. Found: C, 76.6; H, 7.98; N, 4.68. The enantiomeric excess was determined using CHIRALCEL OD-R column, $\mathrm{CH}_{3} \mathrm{CN} / 1 \mathrm{M} \mathrm{NaClO}{ }_{4}$ aq $=35 / 65$, flow rate $=0.8 \mathrm{~mL} / \mathrm{min}$, UV detection at $254 \mathrm{~nm}, \mathrm{t}_{\mathrm{R}}=31.9 \min$ (major enantiomer), $\mathrm{t}_{\mathrm{R}}=36.22 \mathrm{~min}$ (minor enantiomer); $85 \%$ ee. 
N-(4-Methoxybenzyl)-1-(4-methylphenyl)-3-butenylamine, 11c

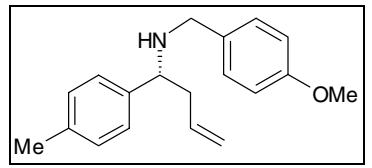

Colorless oil; $[\alpha]_{\mathrm{D}}^{22}=+48.5\left(\mathrm{c} 1.0, \mathrm{CHCl}_{3}\right.$ ); IR (neat) 3325, 3002, 2910, 2833, 1638, 1612, 1584, 1512, 1463 ,

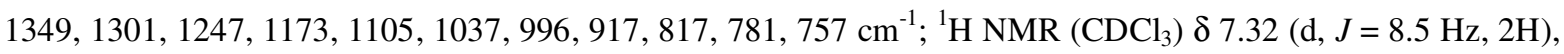
$7.24(\mathrm{~d}, J=8 \mathrm{~Hz}, 4 \mathrm{H}), 6.92(\mathrm{~d}, J=8.5 \mathrm{~Hz}, 2 \mathrm{H}), 5.78(\mathrm{ddt}, J=16 \mathrm{~Hz}, 10.2 \mathrm{~Hz}, 6.8 \mathrm{~Hz}, 1 \mathrm{H}), 5.07-5.15(\mathrm{~m}, 2 \mathrm{H})$, $3.85(\mathrm{~s}, 3 \mathrm{H}), 3.72-3.7(\mathrm{~m}, 1 \mathrm{H}), 3.69(\mathrm{~d}, J=13 \mathrm{~Hz}, 1 \mathrm{H}), 3.53(\mathrm{~d}, J=13 \mathrm{~Hz}, 1 \mathrm{H}), 2.47-2.45(\mathrm{~m}, 2 \mathrm{H}), 2.42(\mathrm{~s}, 3 \mathrm{H})$, 1.73 (brs, $1 \mathrm{H})$;

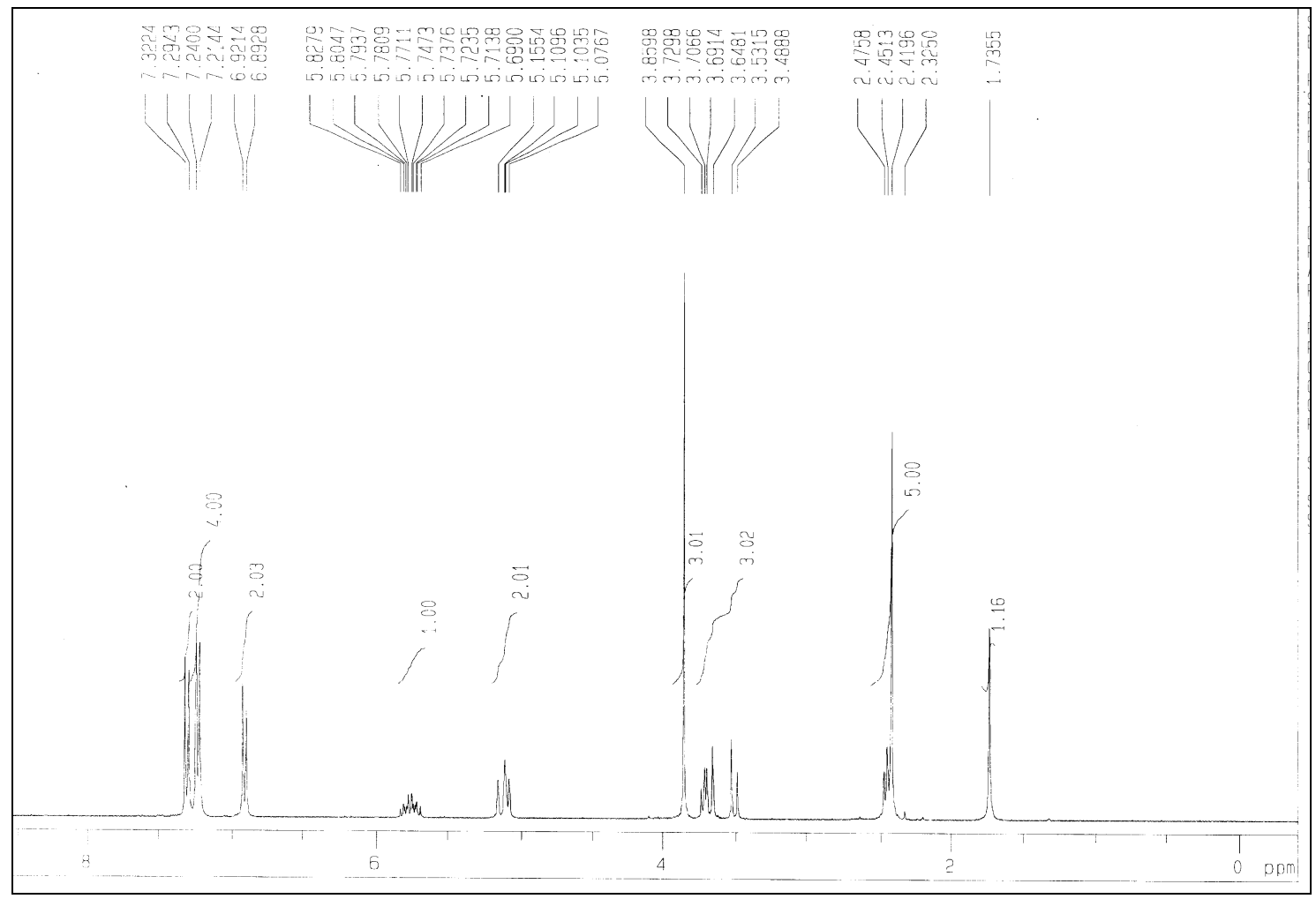

${ }^{13} \mathrm{C}$ NMR $\left(\mathrm{CDCl}_{3}\right) \delta$ 158.4, 140.7, 136.4, 135.6, 132.7, 129.2, 129.0, 127.1, 117.4, 113.6, 61.0, 55.1, 50.6, 43.1, 21.0; MS (EI) $\mathrm{m} / z$ (relative intensity) 240 [M+-allyl] (13.3), 160 (4.9), 121 (100), 119 (4.9), 91 (12.4), 77 (5.3); Anal. Calcd for $\mathrm{C}_{19} \mathrm{H}_{23} \mathrm{NO}$ (281.39): C, 81.10; H, 8.24; N, 4.97. Found: C, 81.02; H, 8.47; N, 4.97. The enantiomeric excess was determined using CHIRALCEL OD-R column, $\mathrm{CH}_{3} \mathrm{CN} / 1 \mathrm{M} \mathrm{NaClO}$ aq $=35 / 65$, flow rate $=0.8 \mathrm{~mL} / \mathrm{min}$, $\mathrm{UV}$ detection at $254 \mathrm{~nm}, \mathrm{t}_{\mathrm{R}}=41.41 \mathrm{~min}$ (major enantiomer), $\mathrm{t}_{\mathrm{R}}=46.64 \mathrm{~min}$ (minor enantiomer); $86 \%$ ee. 
N-(4-Methoxybenzyl)-1-(4-nitrophenyl)-3-butenylamine, 11d

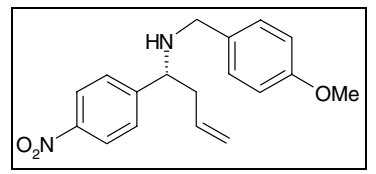

Pale yellow oil; $[\alpha]_{\mathrm{D}}^{22}=+26.9\left(\mathrm{c} 1.0, \mathrm{CHCl}_{3}\right)$; IR (neat) 3331, 3075, 3001, 2933, 2835, 1639, 1609, 1515, 1463,

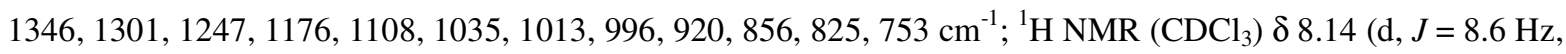
2H), $7.48(\mathrm{~d}, J=8.6 \mathrm{~Hz}, 2 \mathrm{H}), 7.07(\mathrm{~d}, J=8.6 \mathrm{~Hz}, 2 \mathrm{H}), 6.78(\mathrm{~d}, J=8.6 \mathrm{~Hz}, 2 \mathrm{H}), 5.62(\mathrm{ddt}, J=16.4 \mathrm{~Hz}, 10.1 \mathrm{~Hz}$, $6.4 \mathrm{~Hz}, 1 \mathrm{H}), 5.52-4.96(\mathrm{~m}, 2 \mathrm{H}), 3.74-3.73(\mathrm{~m}, 1 \mathrm{H}), 3.72(\mathrm{~s}, 3 \mathrm{H}), 3.52(\mathrm{~d}, J=13 \mathrm{~Hz}, 1 \mathrm{H}), 3.38(\mathrm{~d}, J=13 \mathrm{~Hz}$, 1H), 2.37-2.21 (m, 2H), 1.7 (brs, 1H);

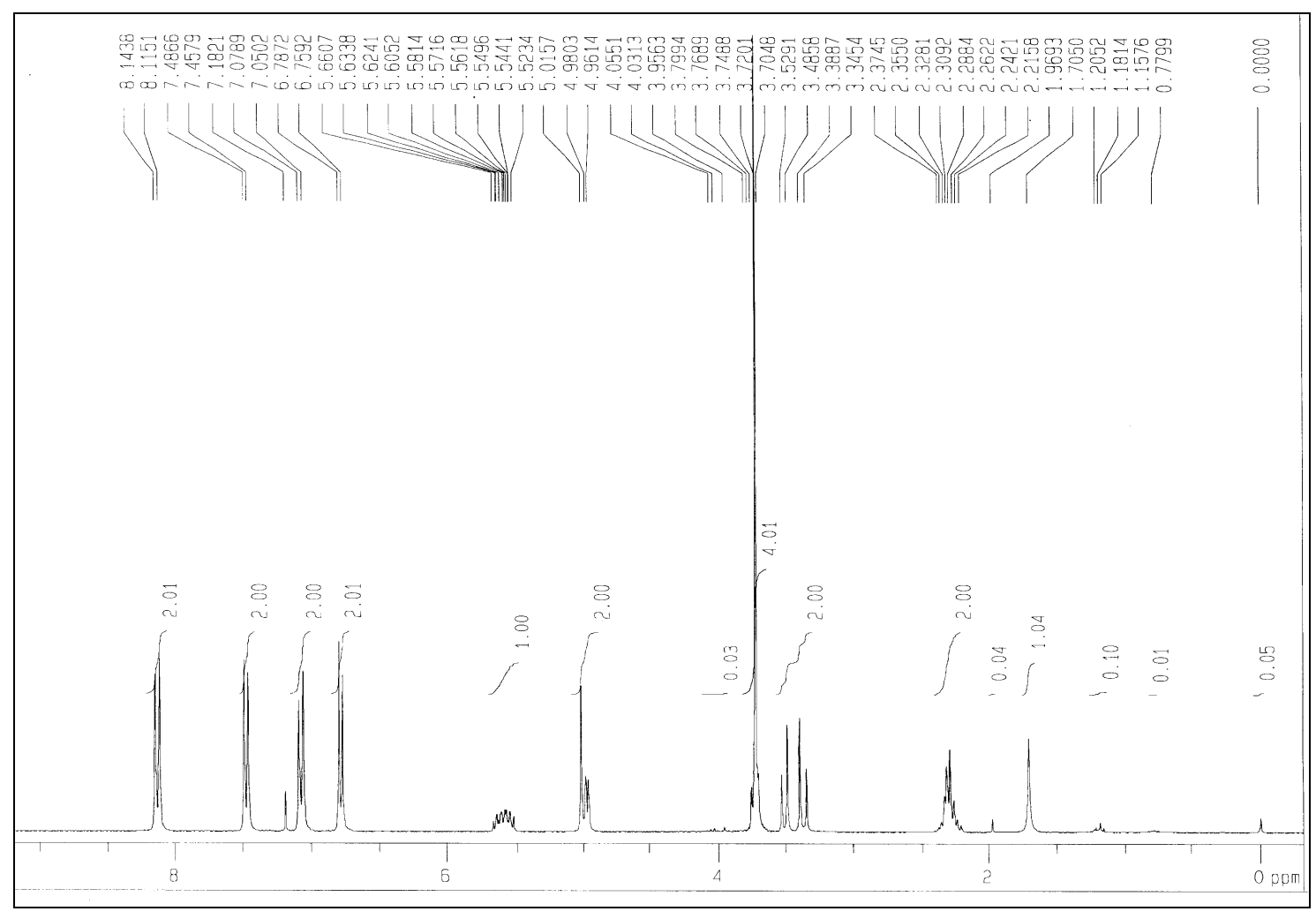

${ }^{13} \mathrm{C} \mathrm{NMR}\left(\mathrm{CDCl}_{3}\right) \delta 158.6,151.8,147.0,134.2,131.9,129.1,128.0,123.6,118.5,113.7,60.9,55.2,50.9,42.8$; MS (EI) $\mathrm{m} / z$ (relative intensity) $271\left[\mathrm{M}^{+}\right.$-allyl] (2), 186 (14.6), 150 (4.3), 121 (44.6), 91 (100), 81 (10.2), 77 (11.8); Anal. Calcd for $\mathrm{C}_{18} \mathrm{H}_{20} \mathrm{~N}_{2} \mathrm{O}_{3}$ (312.37): C, 69.21; H, 6.45; N, 8.96. Found: C, 68.98; H, 6.73; N, 8.8. The enantiomeric excess was determined by converting to trifluoroacetylamide form, $[\alpha]_{\mathrm{D}}^{22}=+30.6\left(\mathrm{c} 1.5, \mathrm{CHCl}_{3}\right)$ and using CHIRALCEL OD column, hexane $/ i$-PrOH $=50 / 1$, flow rate $=0.8 \mathrm{~mL} / \mathrm{min}$, UV detection at $254 \mathrm{~nm}, \mathrm{t}_{\mathrm{R}}$ $=27.43 \min$ (minor enantiomer), $\mathrm{t}_{\mathrm{R}}=29.75 \mathrm{~min}$ (major enantiomer); $42 \%$ ee. 


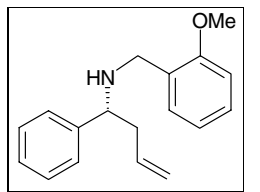

Colorless oil; $[\alpha]_{\mathrm{D}}^{22}=+51.6\left(\mathrm{c} 1.0, \mathrm{CHCl}_{3}\right)$; IR (neat) 3343, 3062, 3026, 3001, 2934, 2835, 1638, 1601, 1588, $\left.1492,1463,1358,1288,1241,1175,1117,1048,1029,955,916,836,754,702 \mathrm{~cm}^{-1} ;{ }^{1} \mathrm{H} \mathrm{NMR}_{(\mathrm{CDCl}}\right) \delta$ 7.34-7.18 (m, 6H), 7.1-7.05 (m, 1H), 6.89-6.82 (m, 2H), 5.66 (ddt, J=16.8 Hz, 10.1 Hz, 6.8 Hz, $1 \mathrm{H}), 5.07$ (ddt, $J=16.8 \mathrm{~Hz}, 2.2 \mathrm{~Hz}, 2 \mathrm{~Hz}, 1 \mathrm{H}), 5.02(\mathrm{ddt}, J=10.1 \mathrm{~Hz}, 2.2 \mathrm{~Hz}, 2 \mathrm{~Hz}, 1 \mathrm{H}), 3.8(\mathrm{~s}, 3 \mathrm{H}), 3.72(\mathrm{~d}, J=13.4 \mathrm{~Hz}, 1 \mathrm{H})$, 3.63-3.6 (m, 1H), $3.58(\mathrm{~d}, J=13.4 \mathrm{~Hz}, 1 \mathrm{H}), 2.39-2.35(\mathrm{~m}, 2 \mathrm{H}), 2.12-1.91$ (brs, $1 \mathrm{H})$;

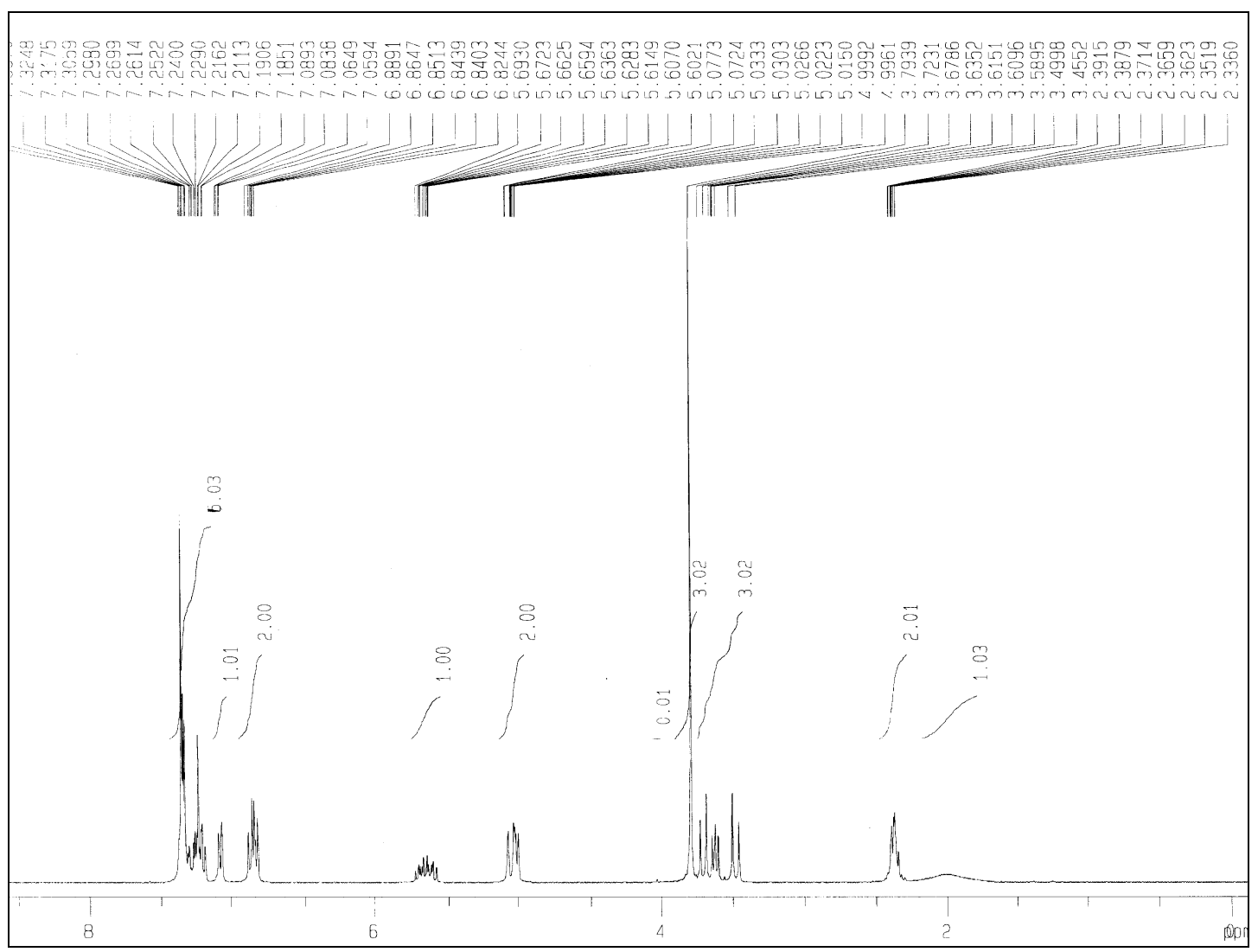

${ }^{13} \mathrm{C} \mathrm{NMR}\left(\mathrm{CDCl}_{3}\right) \delta 157.6,143.8,135.5,129.8,128.2,128.1,128.0,127.3,126.8,120.1,117.3,110.0,60.9,54.9$, 47.1, 43.1; MS (EI) m/z (relative intensity) 226 [M+-allyl] (11.9), 160 (3.9), 122 (9.2), 121 (100), 105 (2.3), 94 (3.1), 93 (37.6), 91 (7.2), 79 (1.3), 77 (1); Anal. Calcd for $\mathrm{C}_{18} \mathrm{H}_{21} \mathrm{NO}$ (267.37): C, 80.86; H, 7.91; N, 5.23. Found: C, 80.83; H, 8.18; N, 5.2. The enantiomeric excess was determined using CHIRALCEL OD-R column, $\mathrm{CH}_{3} \mathrm{CN} / 1 \mathrm{M} \mathrm{NaClO}_{4} \mathrm{aq}=50 / 50$, flow rate $=0.8 \mathrm{~mL} / \mathrm{min}, \mathrm{UV}$ detection at $254 \mathrm{~nm}, \mathrm{t}_{\mathrm{R}}=10.03$ min $($ minor enantiomer), $\mathrm{t}_{\mathrm{R}}=12.66 \mathrm{~min}$ (major enantiomer); $89 \%$ ee. 
N-Benzyl-1-(2-naphthyl)-3-butenylamine, 21a

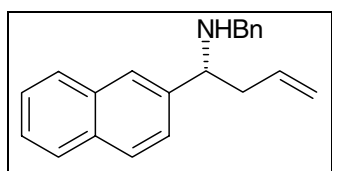

Colorless oil; $[\alpha]_{\mathrm{D}}{ }^{21}=+64.0\left(\mathrm{c} 1.5, \mathrm{CHCl}_{3}\right)$; IR, ${ }^{1} \mathrm{H}$ NMR, ${ }^{13} \mathrm{C}$ NMR, HRMS and analysis data (see ref. 2). The enantiomeric excess was determined by using CHIRALCEL OD-R column, $\mathrm{CH}_{3} \mathrm{CN} / 1 \mathrm{M} \mathrm{NaClO}$ aq $=40 / 60$, flow rate $=0.8 \mathrm{~mL} / \mathrm{min}$, $\mathrm{UV}$ detection at $254 \mathrm{~nm}, \mathrm{t}_{\mathrm{R}}=58.3 \mathrm{~min}$ (major enantiomer), $\mathrm{t}_{\mathrm{R}}=67.45 \mathrm{~min}$ (minor enantiomer); $91 \%$ ee.

N-(4-Methoxybenzyl)-1-(2-naphthyl)-3-butenylamine, 21b

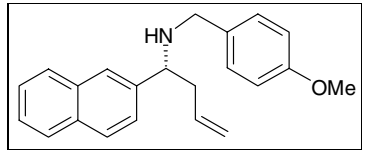

Colorless oil; $[\alpha]_{\mathrm{D}}^{22}=+54.3\left(\mathrm{c} 1.0, \mathrm{CHCl}_{3}\right)$; IR (neat) 3330, 3076, 3026, 2978, 2911, 2833, 1640, 1602, 1492, $1454,1415,1357,1115,1070,1027,994,759 \mathrm{~cm}^{-1} ;{ }^{1} \mathrm{H}$ NMR $\left(\mathrm{CDCl}_{3}\right) \delta$ 7.95-7.86 (m, 4H), 7.63-7.51 (m, 3H), $7.26(\mathrm{~d}, J=8.6 \mathrm{~Hz}, 2 \mathrm{H}), 6.94(\mathrm{~d}, J=8.6 \mathrm{~Hz}, 2 \mathrm{H}), 5.85(\mathrm{ddt}, J=16.4 \mathrm{~Hz}, 10.2 \mathrm{~Hz}, 6.9 \mathrm{~Hz}, 1 \mathrm{H}), 5.2-5.1(\mathrm{~m}, 2 \mathrm{H})$, $3.95-3.91(\mathrm{~m}, 1 \mathrm{H}), 3.87(\mathrm{~s}, 3 \mathrm{H}), 3.74(\mathrm{~d}, J=13 \mathrm{~Hz}, 1 \mathrm{H}), 3.5(\mathrm{~d}, J=13 \mathrm{~Hz}, 1 \mathrm{H}), 2.62-2.52(\mathrm{~m}, 2 \mathrm{H}), 1.87$ (brs, $1 \mathrm{H})$;

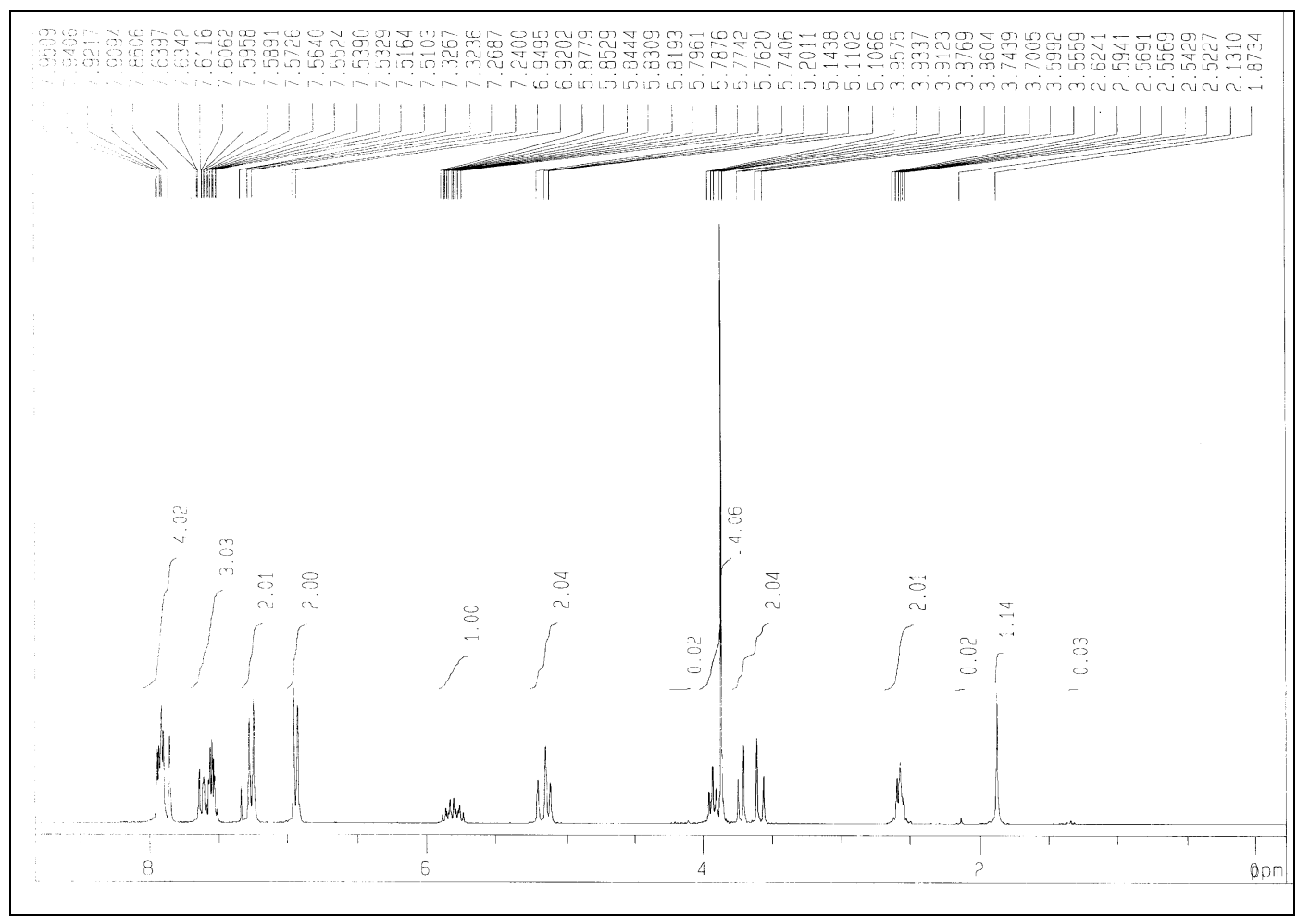


${ }^{13} \mathrm{C}$ NMR $\left(\mathrm{CDCl}_{3}\right) \delta 158.4,141.1,135.3,133.3,132.7,132.5,129.1,128.0,127.6,126.1,125.8,125.3,125.2$, 117.5, 113.6, 61.4, 55.0, 50.6, 42.8; MS (EI) $\mathrm{m} / z$ (relative intensity) 276 [ $\mathrm{M}^{+}$-allyl] (7.7), 160 (6.6), 122 (8.7), 121 (100), 91 (4.8), 77 (2.4); Anal. Calcd for $\mathrm{C}_{22} \mathrm{H}_{23} \mathrm{NO}$ (317.43): C, 83.24; H, 7.3; N, 4.41. Found: C, 83.19; H, 7.49; $\mathrm{N}, 4.58$. The enantiomeric excess was determined using CHIRALCEL OD-R column, $\mathrm{CH}_{3} \mathrm{CN} / 1 \mathrm{M} \mathrm{NaClO} 4$ $\mathrm{aq}=50 / 50$, flow rate $=0.7 \mathrm{~mL} / \mathrm{min}, \mathrm{UV}$ detection at $254 \mathrm{~nm}, \mathrm{t}_{\mathrm{R}}=38.16 \mathrm{~min}$ (major enantiomer), $\mathrm{t}_{\mathrm{R}}=42.87 \mathrm{~min}$ (minor enantiomer); $82 \%$ ee.

\section{N-allyl-1-phenyl-3-butenylamine, 23a}

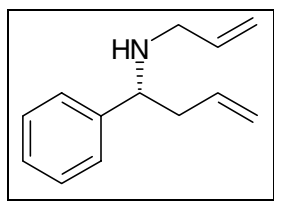

Colorless oil; $[\alpha]_{\mathrm{D}}^{23}=+24.8\left(\mathrm{c} 1.0, \mathrm{CHCl}_{3}\right)$; IR (neat) 3329, 3077, 3026, 3003, 2977, 2910, 2833, 1640, 1602, 1542, 1492, 1454, 1357, 1307, 1235, 1199, 1069, 1027, 994, 917, $758 \mathrm{~cm}^{-1} ;{ }^{1} \mathrm{H}$ NMR $\left(\mathrm{CDCl}_{3}\right) \delta 7.35-7.23(\mathrm{~m}$, $5 \mathrm{H}), 5.86-5.73(\mathrm{~m}, 1 \mathrm{H}), 5.73-5.7(\mathrm{~m}, 1 \mathrm{H}), 5.14-5.03(\mathrm{~m}, 4 \mathrm{H}), 3.72(\mathrm{dd}, J=6.2 \mathrm{~Hz}, 1.2 \mathrm{~Hz}, 1 \mathrm{H}), 3.11-3.02(\mathrm{~m}$, $2 \mathrm{H}), 2.44-2.39(\mathrm{~m}, 2 \mathrm{H}), 1.71$ (brs, $1 \mathrm{H})$;

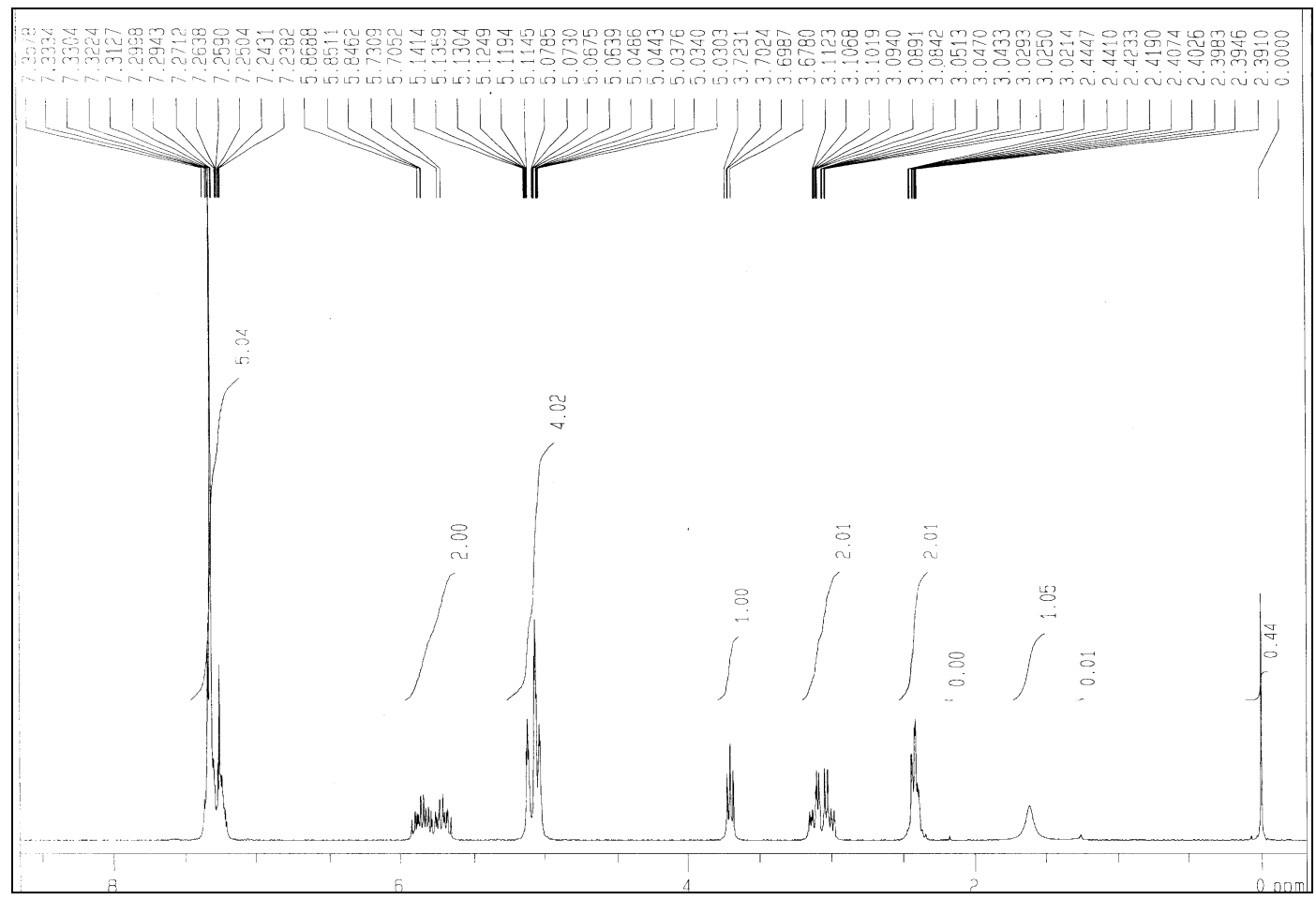

${ }^{13} \mathrm{C}$ NMR $\left(\mathrm{CDCl}_{3}\right) \delta 143.4,136.4,135.2,128.5,126.9,126.7,117.3,115.4,61.4,49.7,42.8 ; \mathrm{MS}(\mathrm{EI}) \mathrm{m} / z$ (relative intensity) 188 [M+1] (0.9), 146 [M+-allyl] (16.1), 129 (14.9), 119 (2.6), 104 (4.8), 91 (100), 77 (2.1); Anal. Calcd for $\mathrm{C}_{13} \mathrm{H}_{17} \mathrm{~N}$ (187.28): C, 83.37; H, 9.14; N, 7.47. Found: C, 83.07; H, 9.35; N, 7.4. The enantiomeric excess was determined by converting to trifluoroacetylamide form, $[\alpha]_{\mathrm{D}}{ }^{22}=+93.2\left(\mathrm{c} 1.0, \mathrm{CHCl}_{3}\right)$ 
and using CHIRALCEL OD column, hexane $/ i-\mathrm{PrOH}=300 / 1$, flow rate $=0.6 \mathrm{~mL} / \mathrm{min}$, UV detection at $254 \mathrm{~nm}$, $t_{R}=16.17 \min$ (major enantiomer), $t_{R}=17.85 \min$ (minor enantiomer); $78 \%$ ee.

\section{N-Methyl-1-(3-pyridyl)-3-butenylamine, 25}

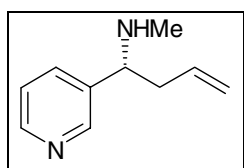

Yellow liquid; $[\alpha]_{\mathrm{D}}^{22}=+43.1\left(\mathrm{c} 1.66, \mathrm{CHCl}_{3}\right)$; IR (neat) 3324, 3082, 2979, 2936, 1666, 1640, 1578, 1476, 1428, 1098, 1027, 996, $734 \mathrm{~cm}^{-1} ;{ }^{1} \mathrm{H}$ NMR $\left(\mathrm{CDCl}_{3}\right) \delta 8.45-8.41(\mathrm{~m}, 2 \mathrm{H}), 7.62-7.58(\mathrm{~m}, 1 \mathrm{H}), 7.23-7.2(\mathrm{~m}, 1 \mathrm{H})$, 5.69-5.59 (m, 1H), 5.08-5.01 (m, 2H), 3.64-3.60 (m, 1H), $3.51(\mathrm{t}, J=6.8 \mathrm{~Hz}, 1 \mathrm{H}), 2.47-2.4(\mathrm{~m}, 2 \mathrm{H}), 2.2(\mathrm{~s}$, $3 \mathrm{H})$;

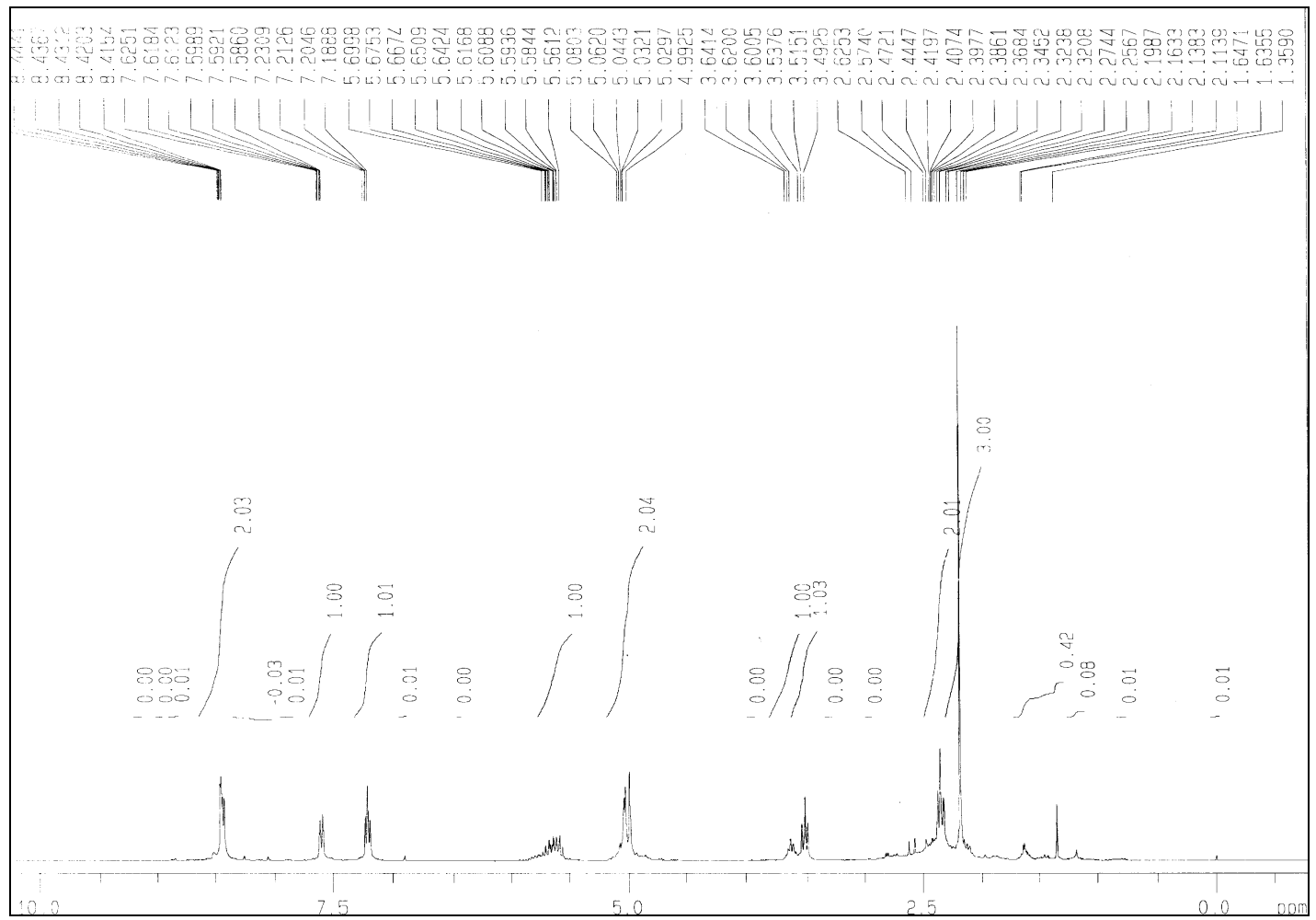

${ }^{13} \mathrm{C} \mathrm{NMR}\left(\mathrm{CDCl}_{3}\right) \delta 149.2,148.5,138.6,134.7,134.3,123.5,118.3,62.1,42.4,34.7 ; \mathrm{MS}$ (EI) $\mathrm{m} / z$ (relative intensity) $163\left[\mathrm{M}^{+}+\mathrm{H}\right]$ (3.5), $161\left[\mathrm{M}^{+}-\mathrm{H}\right]$ (6.3), $121\left[\mathrm{M}^{+}\right.$-allyl] (100), 119 (9.6), 94 (92), 80 (18.7), 78 (9.4); Anal. Calcd for $\mathrm{C}_{10} \mathrm{H}_{14} \mathrm{~N}_{2}$ (162.23): C, 74.04; H, 8.69; N, 17.27. Found: C, 74.24; H, 8.85; N, 16.94. The enantiomeric excess was determined by converting to trifluoroacetylamide form, $[\alpha]_{\mathrm{D}}^{22}=+90.5\left(\mathrm{c} 0.5, \mathrm{CHCl}_{3}\right)$ and using CHIRALCEL OD column, hexane $/ i-\mathrm{PrOH}=10 / 1$, flow rate $=0.7 \mathrm{~mL} / \mathrm{min}$, UV detection at $254 \mathrm{~nm}, \mathrm{t}_{\mathrm{R}}$ $=24.13 \min$ (minor enantiomer), $\mathrm{t}_{\mathrm{R}}=32.58 \mathrm{~min}$ (major enantiomer); $55 \%$ ee. 
N-Benzyl-1-(3,4,5-trimethoxyphenyl)-3-butenylamine, 27

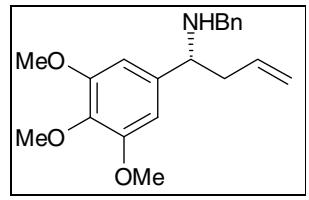

Colorless syrupy liquid; $[\alpha]_{\mathrm{D}}^{22}=+15.3\left(\mathrm{c} 1.0, \mathrm{CHCl}_{3}\right)$; IR (neat) $3325,3062,2997,2936,2835,1638,1590$, $1505,1462,1419,1325,1233,1183,1128,1009,917,831,739 \mathrm{~cm}^{-1} ;{ }^{1} \mathrm{H}$ NMR $\left(\mathrm{CDCl}_{3}\right) \delta 7.34-7.21(\mathrm{~m}, 5 \mathrm{H}), 6.6$ (s, 2H), 5.73 (ddt, $J=16.6 \mathrm{~Hz}, 10.0 \mathrm{~Hz}, 6.9 \mathrm{~Hz}, 1 \mathrm{H}), 5.13-5.05$ (m, 2H), 3.87 (s, 6H), 3.85 (s, 3H), 3.71 (d, $J=$ $13.2 \mathrm{~Hz}, 1 \mathrm{H}), 3.63-3.59(\mathrm{~m}, 1 \mathrm{H}), 3.56(\mathrm{~d}, J=13.2 \mathrm{~Hz}, 1 \mathrm{H}), 2.45-2.31(\mathrm{~m}, 2 \mathrm{H}), 1.81$ (brs, $1 \mathrm{H})$;

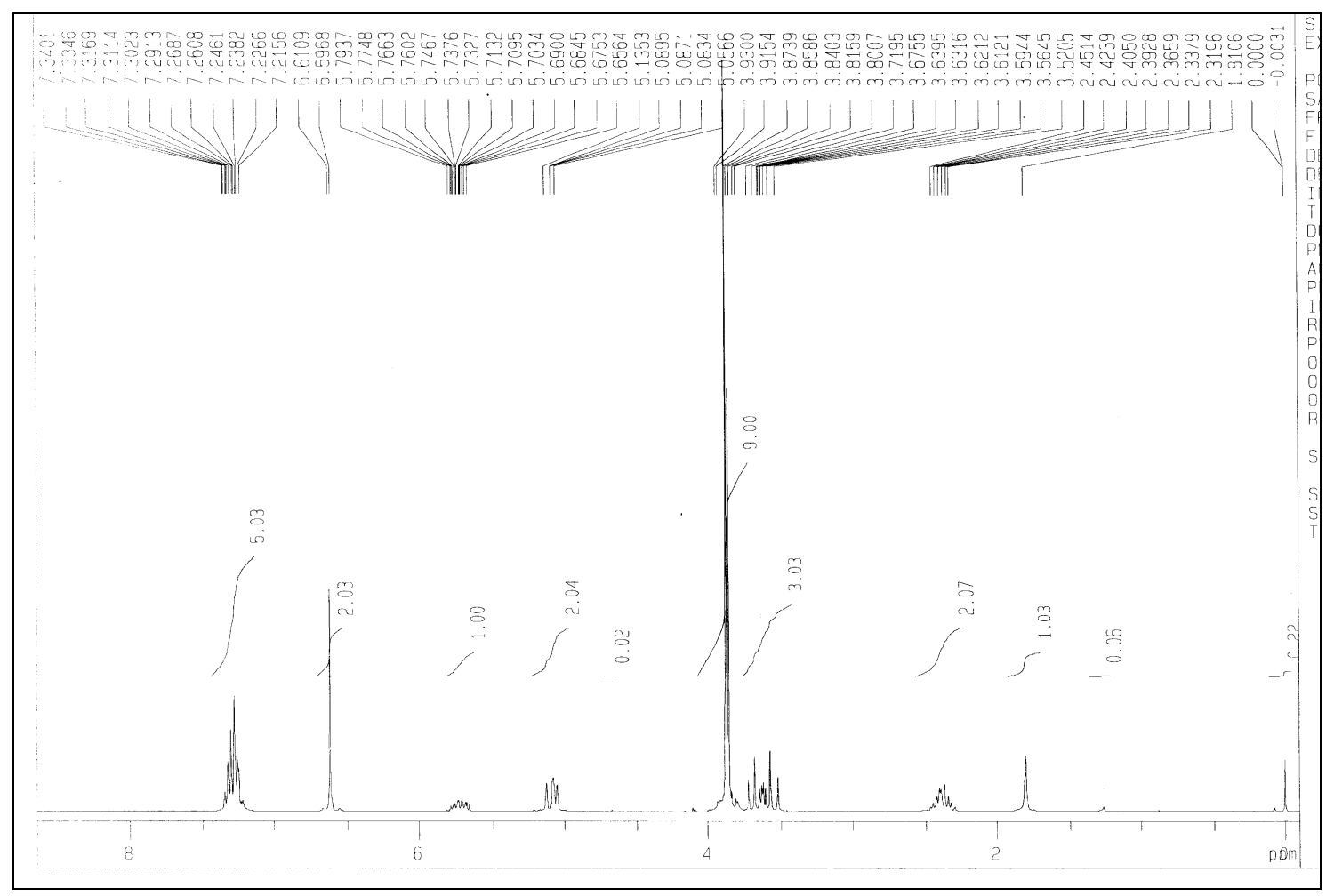

${ }^{13} \mathrm{C}$ NMR $\left(\mathrm{CDCl}_{3}\right) \delta$ 153.2, 140.5, 139.6, 136.6, 135.4, 128.3, 128.1, 126.8, 117.7, 103.8, 61.8, 60.8, 56.0, 51.4, 43.3; MS (EI) $\mathrm{m} / z$ (relative intensity) 286 [M $\mathrm{M}^{+}$-allyl] (14.9), 254 (8.4), 238 (23.2), 209 (6.1), 195 (6.9), 181 (13.8), 169 (4), 125 (1.2), 91 (100); Anal. Calcd for $\mathrm{C}_{20} \mathrm{H}_{25} \mathrm{NO}_{3}$ (327.42): C, 73.36; H, 7.69; N, 4.27. Found: C, 73.12; $\mathrm{H}, 7.89 ; \mathrm{N}, 4.57$. The enantiomeric excess was determined using CHIRALCEL OD-R column, $\mathrm{CH}_{3} \mathrm{CN} / 1 \mathrm{M} \mathrm{NaClO}{ }_{4} \mathrm{aq}=30 / 70$, flow rate $=0.8 \mathrm{~mL} / \mathrm{min}$, $\mathrm{UV}$ detection at $254 \mathrm{~nm}, \mathrm{t}_{\mathrm{R}}=31.15$ min (major enantiomer), $\mathrm{t}_{\mathrm{R}}=34.64 \mathrm{~min}$ (minor enantiomer); $34 \%$ ee. 


\section{N-Benzyl-1-piperonyl-3-butenylamine, 9a}

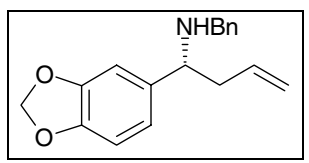

Colorless oil; $[\alpha]_{\mathrm{D}}{ }^{22}=+59.1$ (c 1.0, $\mathrm{CHCl}_{3}$ ); IR (neat) 3327, 3063, 3026, 2976, 2894, 1638, 1607, 1468, 1439, 1376, 1243, 1183, 1100, 1040, 996, 865, $736 \mathrm{~cm}^{-1} ;{ }^{1} \mathrm{H}$ NMR $\left(\mathrm{CDCl}_{3}\right) \delta 7.32-7.21(\mathrm{~m}, 5 \mathrm{H}), 6.91(\mathrm{~s}, 1 \mathrm{H}), 6.77(\mathrm{~s}$, 2H), 5.95 (s, 2H), 5.69 (ddt, $J=17.0 \mathrm{~Hz}, 10.2 \mathrm{~Hz}, 7.2 \mathrm{~Hz}, 1 \mathrm{H}$ ), 5.09 (ddt, $J=17.0 \mathrm{~Hz}, 2.2 \mathrm{~Hz}, 2 \mathrm{~Hz}, 1 \mathrm{H}$ ), 5.03 (ddt, $J=10.2 \mathrm{~Hz}, 2.2 \mathrm{~Hz}, 2 \mathrm{~Hz}, 1 \mathrm{H}), 3.66(\mathrm{~d}, J=13.2 \mathrm{~Hz}, 1 \mathrm{H}), 3.6(\mathrm{t}, J=6.8 \mathrm{~Hz}, 1 \mathrm{H}), 3.51(\mathrm{~d}, J=13.2 \mathrm{~Hz}, 1 \mathrm{H})$, $2.38-2.28(\mathrm{~m}, 2 \mathrm{H}), 1.7$ (brs, $1 \mathrm{H})$;

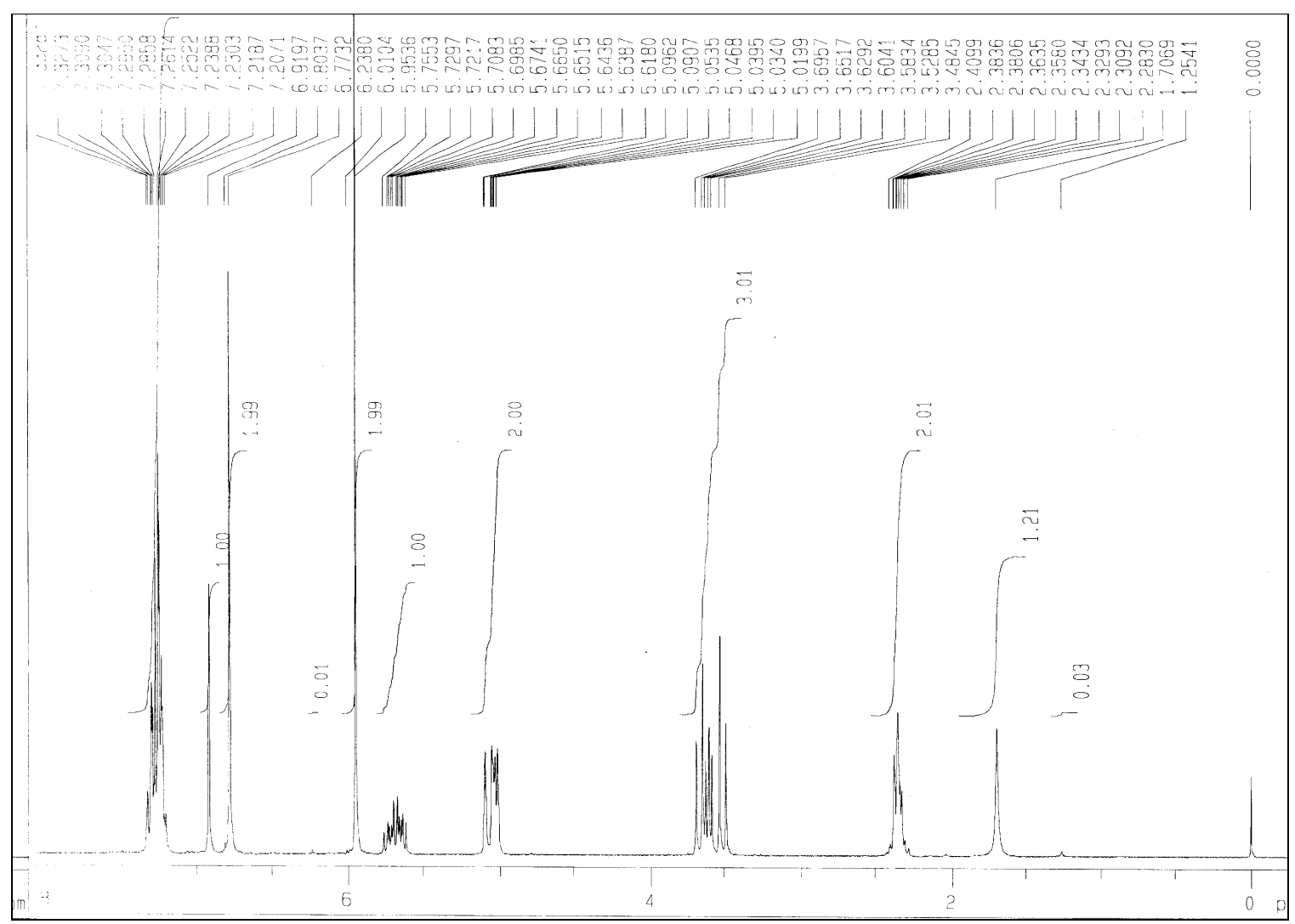

${ }^{13} \mathrm{C} \mathrm{NMR}\left(\mathrm{CDCl}_{3}\right) \delta 147.8,146.4,140.5,137.8,135.3,128.3,128.0,126.8,120.5,117.5,107.9,107.2,100.8$, 61.3, 51.3, 43.2; MS (EI) m/z (relative intensity) 240 [ $\mathrm{M}^{+}$-allyl] (100), 148 (3.9), 117 (2.5), 91 (81), 65 (5.7); Anal. Calcd for $\mathrm{C}_{18} \mathrm{H}_{19} \mathrm{NO}_{2}$ (281.35): C, 76.84; H, 6.8; N, 4.97. Found: C, 76.52; H, 6.68; N, 5.21. The enantiomeric excess was determined using CHIRALCEL OD-R column, $\mathrm{CH}_{3} \mathrm{CN} / 1 \mathrm{M} \mathrm{NaClO}$ aq $=40 / 60$, flow rate $=0.8 \mathrm{~mL} / \mathrm{min}, \mathrm{UV}$ detection at $254 \mathrm{~nm}, \mathrm{t}_{\mathrm{R}}=18.4 \mathrm{~min}$ (major enantiomer), $\mathrm{t}_{\mathrm{R}}=22.2 \mathrm{~min}$ (minor enantiomer); $90 \%$ ee. 
N-(4-Methoxybenzyl)-1-piperonyl-3-butenylamine, 9b

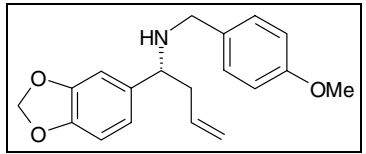

Colorless oil; $[\alpha]_{\mathrm{D}}{ }^{22}=+54.9\left(\mathrm{c} 1.0, \mathrm{CHCl}_{3}\right.$ ); IR (neat) 3327, 3072, 2902, 2834, 1638, 1611, 1584, 1512, 1376, 1300, 1244, 1177, 1101, 1038, 997, 814, $759 \mathrm{~cm}^{-1} ;{ }^{1} \mathrm{H}$ NMR $\left(\mathrm{CDCl}_{3}\right) \delta 7.26(\mathrm{~d}, J=8.4 \mathrm{~Hz}, 2 \mathrm{H}), 7.0(\mathrm{~s}, 1 \mathrm{H}), 6.95$ (d, $J=8.4 \mathrm{~Hz}, 2 \mathrm{H}), 6.85$ (s, 2H), 6.04 (s, 2H), 5.78 (ddt, $J=16.1 \mathrm{~Hz}, 10.1 \mathrm{~Hz}, 6.6 \mathrm{~Hz}, 1 \mathrm{H}), 5.18$ (ddt, $J=16.1$ Hz, $2.1 \mathrm{~Hz}, 2 \mathrm{~Hz}, 1 \mathrm{H}), 5.12$ (ddt, $J=10.1 \mathrm{~Hz}, 2.1 \mathrm{~Hz}, 2 \mathrm{~Hz}, 1 \mathrm{H}), 3.87$ (s, 3H), 3.71-3.7 (m, 1H), 3.67 (d, $J=13$ $\mathrm{Hz}, 1 \mathrm{H}), 3.55(\mathrm{~d}, J=13 \mathrm{~Hz}, 1 \mathrm{H}), 2.46-2.42(\mathrm{~m}, 2 \mathrm{H}), 1.77$ (brs, $1 \mathrm{H})$;

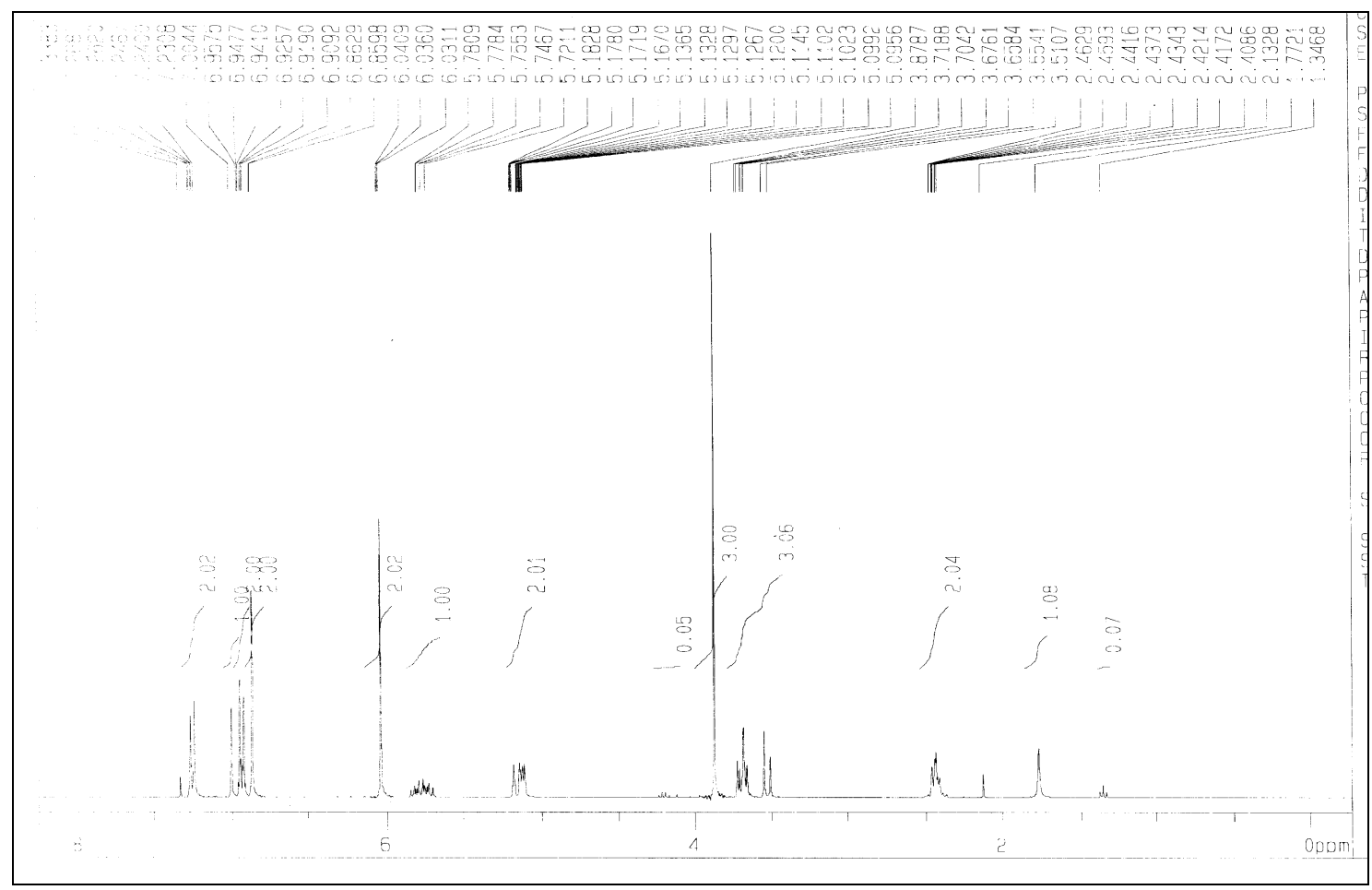

${ }^{13} \mathrm{C}$ NMR $\left(\mathrm{CDCl}_{3}\right) \delta 158.4,147.7,146.4,137.8,135.4,132.6,129.2,120.5,117.4,113.6,107.8,107.2,100.8$, 61.1, 55.2, 50.6, 43.1; MS (EI) m/z (relative intensity) 270 [M+-allyl] (8.4), 160 (5.4), 122 (9.8), 121 (100), 91 (7.4), 77 (2.8); Anal. Calcd for $\mathrm{C}_{19} \mathrm{H}_{21} \mathrm{NO}_{3}$ (311.38): C, 73.28; H, 6.79; N, 4.49. Found: C, 73.07; H, 6.9; N, 4.78. The enantiomeric excess was determined using CHIRALCEL OD-R column, $\mathrm{CH}_{3} \mathrm{CN} / 1 \mathrm{M} \mathrm{NaClO} 4 \mathrm{aq}=35 / 65$, flow rate $=0.8 \mathrm{~mL} / \mathrm{min}$, $\mathrm{UV}$ detection at $254 \mathrm{~nm}, \mathrm{t}_{\mathrm{R}}=34.37 \min$ (major enantiomer), $\mathrm{t}_{\mathrm{R}}=38.25 \mathrm{~min}$ (minor enantiomer); $89 \%$ ee. 


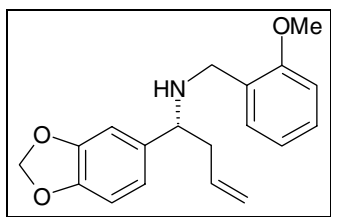

Colorless oil; $[\alpha]_{\mathrm{D}}{ }^{22}=+57.8$ (c 1.0, $\mathrm{CHCl}_{3}$ ); IR (neat) 3341, 3073, 3001, 2902, 2834, 1638, 1601, 1588, 1490, 1376, 1326, 1289, 1180, 1098, 996, 868, $755 \mathrm{~cm}^{-1} ;{ }^{1} \mathrm{H}$ NMR $\left(\mathrm{CDCl}_{3}\right) \delta$ 7.18-7.12 (m, 1H), 7.03-7.0 (m, 1H), 6.85-6.76 (m, 3H), 6.69 (s, 2H), 5.87 (s, 2H), 5.6 (ddt, $J=16.4 \mathrm{~Hz}, 10.2 \mathrm{~Hz}, 6.6 \mathrm{~Hz}, 1 \mathrm{H}), 5.0$ (ddt, $J=16.4 \mathrm{~Hz}$, $2.1 \mathrm{~Hz}, 2 \mathrm{~Hz}, 1 \mathrm{H}), 4.96$ (ddt, $J=10.2 \mathrm{~Hz}, 2.1 \mathrm{~Hz}, 2 \mathrm{~Hz}, 1 \mathrm{H}), 3.75$ (s, 3H), 3.65 (d, $J=13.4 \mathrm{~Hz}, 1 \mathrm{H}), 3.48-3.46$ (m, 1H), $3.42(\mathrm{~d}, J=13.4 \mathrm{~Hz}, 1 \mathrm{H}), 2.3-2.17$ (m, 2H), 1.95 (brs, 1H);

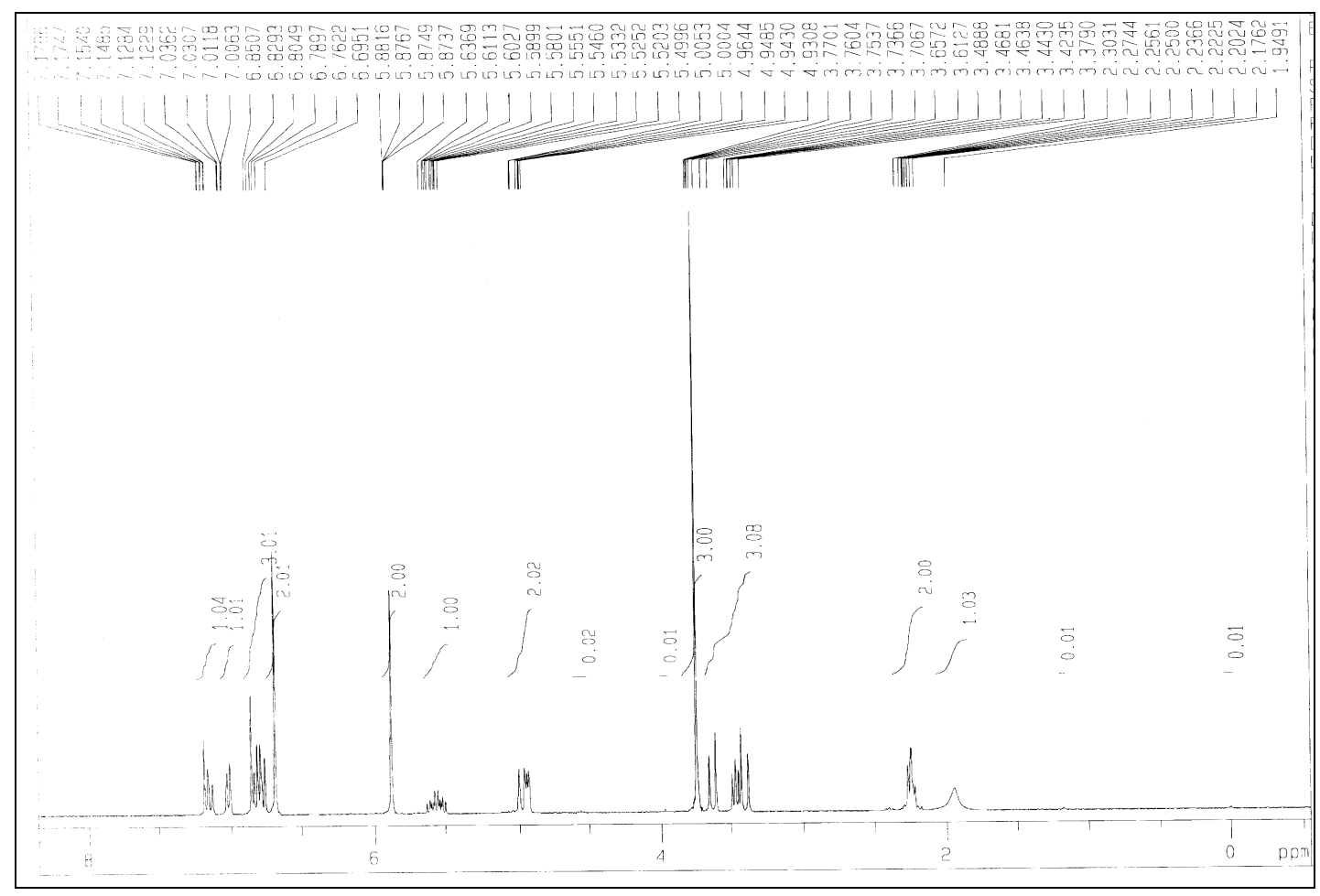

${ }^{13} \mathrm{C}$ NMR $\left(\mathrm{CDCl}_{3}\right) \delta 157.5,147.0,146.2,137.9,135.5,129.8,128.2,128.0,120.5,120.1,117.2,110.0,107.7$, 107.3, 100.6, 60.7, 54.9, 46.9, 43.2; MS (EI) $\mathrm{m} / z$ (relative intensity) 270 [M+-allyl] (10.5), 160 (5.9), 148 (1.4), 122 (8.9), 121 (100), 93 (23), 91 (3.5); Anal. Calcd for $\mathrm{C}_{19} \mathrm{H}_{21} \mathrm{NO}_{3}$ (311.38): C, 73.28; H, 6.79; N, 4.49. Found: $\mathrm{C}$, 73.1; H, 7.01; N, 4.4. The enantiomeric excess was determined using CHIRALCEL OD-R column, $\mathrm{CH}_{3} \mathrm{CN} / 1 \mathrm{M}$ $\mathrm{NaClO}_{4}$ aq $=50 / 50$, flow rate $=0.7 \mathrm{~mL} / \mathrm{min}, \mathrm{UV}$ detection at $254 \mathrm{~nm}, \mathrm{t}_{\mathrm{R}}=13.3 \mathrm{~min}$ (minor enantiomer), $\mathrm{t}_{\mathrm{R}}=$ 17.11 min (major enantiomer); $89 \%$ ee. 


\section{N-Benzyl)-1-(2-chloropiperonyl-3-butenylamine, 9d}

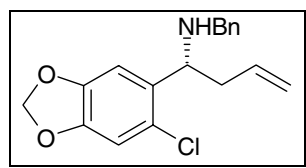

Colorless oil; $[\alpha]_{\mathrm{D}}{ }^{22}=+48.0\left(\mathrm{c} 1.0, \mathrm{CHCl}_{3}\right.$ ); IR (neat) 3330, 3075, 3027, 2977, 2896, 1639, 1603, 1502, 1475, $1408,1391,1375,1269,1237,1159,1110,1038,996,935,841,796,734 \mathrm{~cm}^{-1} ;{ }^{1} \mathrm{H}$ NMR $\left(\mathrm{CDCl}_{3}\right) \delta 7.92-7.21$ (m, 5H), $7.2(\mathrm{~s}, 1 \mathrm{H}), 6.77(\mathrm{~s}, 1 \mathrm{H}), 5.94-5.92(\mathrm{~m}, 2 \mathrm{H}), 5.74(\mathrm{ddt}, J=16.8 \mathrm{~Hz}, 10.2 \mathrm{~Hz}, 6.8 \mathrm{~Hz}, 1 \mathrm{H}), 5.07-5.0(\mathrm{~m}$, 2H), 4.15 (dd, $J=4.8 \mathrm{~Hz}, 3.8 \mathrm{~Hz}, 1 \mathrm{H}), 3.62(\mathrm{~d}, J=13.2 \mathrm{~Hz}, 1 \mathrm{H}), 3.5(\mathrm{~d}, J=13.2 \mathrm{~Hz}, 1 \mathrm{H}), 2.43-2.35(\mathrm{~m}, 1 \mathrm{H})$, $2.23-2.13(\mathrm{~m}, 1 \mathrm{H}), 1.66$ (brs, 1H);

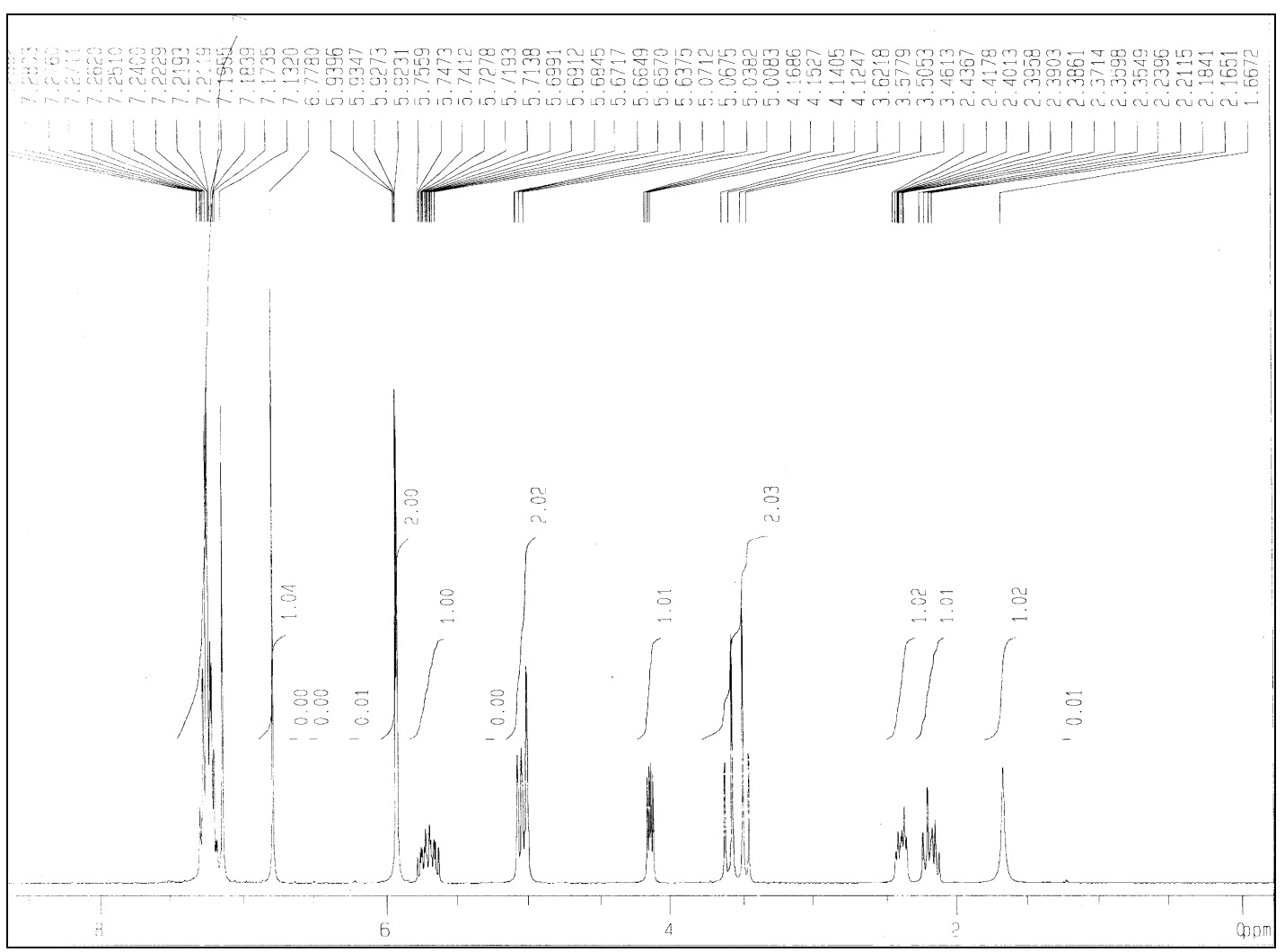

${ }^{13} \mathrm{C}$ NMR $\left(\mathrm{CDCl}_{3}\right) \delta 147.1,146.7,140.3,134.9,134.2,128.2,128.0,126.8,124.9,117.8,109.5,107.4,101.5$, 57.1, 51.4, 41.4; MS (EI) m/z (relative intensity) 274 [M+-allyl] (7.5), 169 (2), 115 (2.2), 91 (100); Anal. Calcd for $\mathrm{C}_{18} \mathrm{H}_{18} \mathrm{ClNO}_{2}$ (315.8): C, 68.46; H, 5.75; N, 4.43; Cl, 11.23. Found: C, 68.4; H, 5.85; N, 4.46; Cl, 11.11. The enantiomeric excess was determined using CHIRALCEL OD-R column, $\mathrm{CH}_{3} \mathrm{CN} / 1 \mathrm{M} \mathrm{NaClO}$ aq $=60 / 40$, flow rate $=0.6 \mathrm{~mL} / \mathrm{min}, \mathrm{UV}$ detection at $254 \mathrm{~nm}, \mathrm{t}_{\mathrm{R}}=17.13 \mathrm{~min}$ (major enantiomer), $\mathrm{t}_{\mathrm{R}}=19.3 \mathrm{~min}$ (minor enantiomer); $87 \%$ ee. 


\section{N-Benzyl-1-(2-bromopiperonyl-3-butenylamine, 9e}

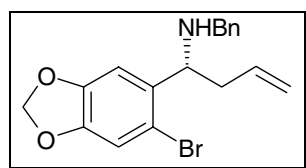

Colorless oil; $[\alpha]_{\mathrm{D}}^{22}=+40.2\left(\mathrm{c} 1.0, \mathrm{CHCl}_{3}\right.$ ); IR (neat) 3330, 3074, 3027, 2976, 2896, 1638, 1604, 1500, 1473, 1406, 1388, 1373, 1234, 1158, 1038, 996, 981, 837, $735 \mathrm{~cm}^{-1} ;{ }^{1} \mathrm{H}$ NMR $\left(\mathrm{CDCl}_{3}\right) \delta 7.38-7.31(\mathrm{~m}, 5 \mathrm{H}), 7.3(\mathrm{~s}$, 1H), $7.03(\mathrm{~s}, 1 \mathrm{H}), 6.02-6.01(\mathrm{~m}, 2 \mathrm{H}), 5.81$ (ddt, $J=16.8 \mathrm{~Hz}, 10.2 \mathrm{~Hz}, 6.7 \mathrm{~Hz}, 1 \mathrm{H}), 5.16-5.09$ (m, 2H), 4.19 (dd, $J=4.6 \mathrm{~Hz}, 3.9 \mathrm{~Hz}, 1 \mathrm{H}), 3.70(\mathrm{~d}, J=13.2 \mathrm{~Hz}, 1 \mathrm{H}), 3.58(\mathrm{~d}, J=13.2 \mathrm{~Hz}, 1 \mathrm{H}), 2.52-2.43(\mathrm{~m}, 1 \mathrm{H}), 2.29-2.18(\mathrm{~m}$, $1 \mathrm{H}), 1.76$ (brs, $1 \mathrm{H})$;

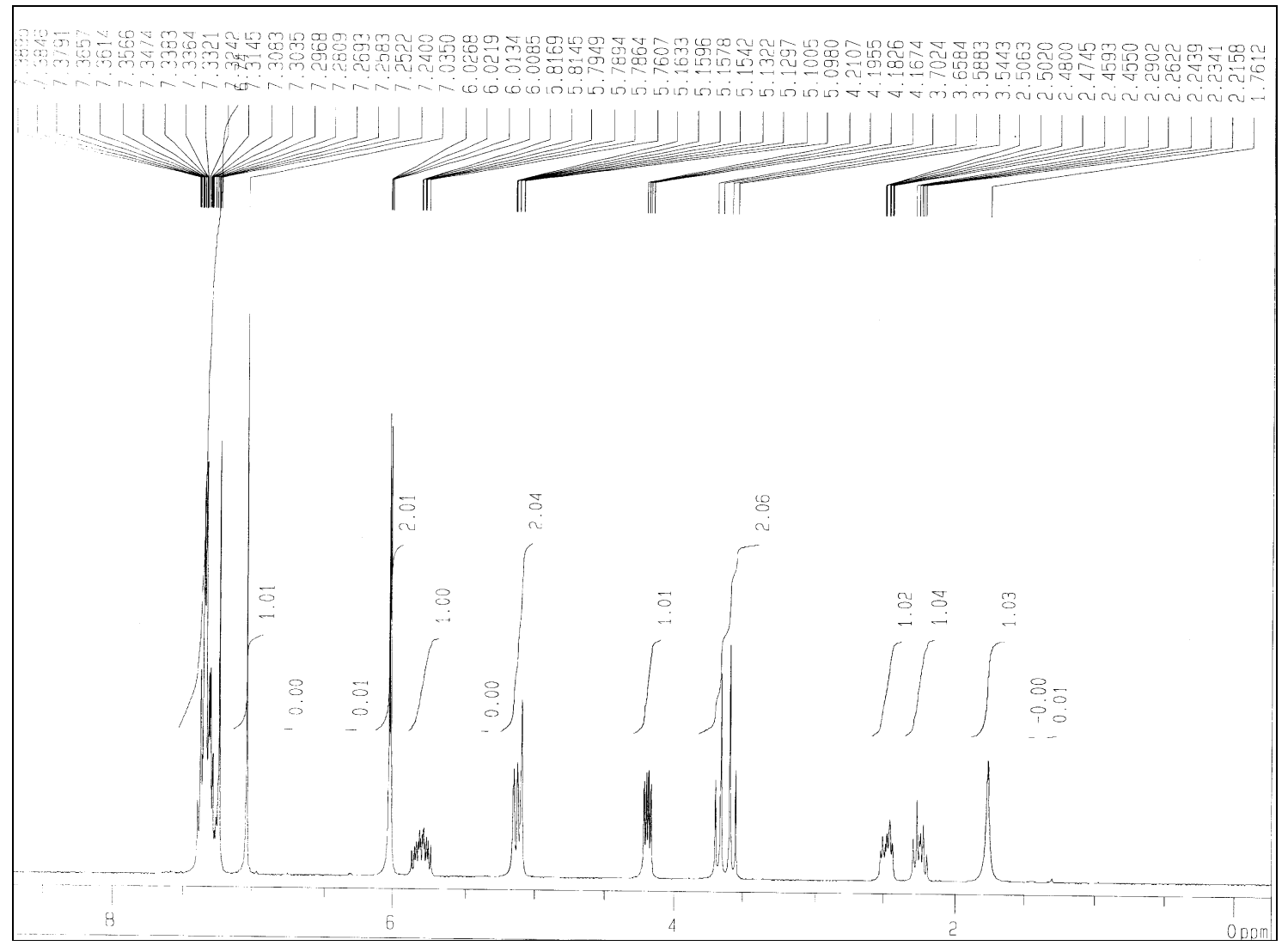

${ }^{13} \mathrm{C} \mathrm{NMR}\left(\mathrm{CDCl}_{3}\right) \delta 147.7,147.0,140.3,135.7,134.9,128.2,128.0,126.8,117.8,113.9,112.4,107.8,101.5$, 59.6, 51.4, 41.5; MS (EI) m/z (relative intensity) 318 [M+-allyl] (1.8), 238 (2.9), 214 (2.1), 148 (2), 107 (2.2), 91 (100); Anal. Calcd for $\mathrm{C}_{18} \mathrm{H}_{18} \mathrm{BrNO}_{2}$ (360.25): C, 60.01; H, 5.04; N, 3.89; Br, 22.18. Found: C, 59.71; H, 5.17; N, 3.98; $\mathrm{Br}, 22.48$. The enantiomeric excess was determined using CHIRALCEL OD-R column, $\mathrm{CH}_{3} \mathrm{CN} / 1 \mathrm{M}$ $\mathrm{NaClO}_{4} \mathrm{aq}=60 / 40$, flow rate $=0.6 \mathrm{~mL} / \mathrm{min}, \mathrm{UV}$ detection at $254 \mathrm{~nm}, \mathrm{t}_{\mathrm{R}}=21.97$ min (major enantiomer), $\mathrm{t}_{\mathrm{R}}=$ $24.92 \mathrm{~min}$ (minor enantiomer); $88 \%$ ee. 
N-Diphenylmethylene-1-piperonyl-3-butenylamine, $9 f$

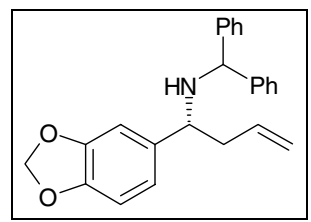

Colorless oil; $[\alpha]_{\mathrm{D}}{ }^{22}=+53.5\left(\mathrm{c} 1.58, \mathrm{CHCl}_{3}\right)$; IR (neat) 3325, 3063, 3027, 2978, 2887, 2775, 1639, 1600, 1547, 1504, 1487, 1445, 1377, 1324, 1246, 1184, 1098, 1075, 1042, 941, 912, 864, $\left.762 \mathrm{~cm}^{-1} ;{ }^{1} \mathrm{H} \mathrm{NMR}_{(\mathrm{CDCl}}\right) \delta$ $7.32-7.13(\mathrm{~m}, 10 \mathrm{H}), 6.81(\mathrm{~d}, J=2 \mathrm{~Hz}, 1 \mathrm{H}), 6.74(\mathrm{~d}, J=8 \mathrm{~Hz}, 1 \mathrm{H}), 6.64(\mathrm{dd}, J=8 \mathrm{~Hz}, 2 \mathrm{~Hz}, 1 \mathrm{H}), 5.92(\mathrm{~s}, 2 \mathrm{H})$, 5.65 (ddt, $J=16.8 \mathrm{~Hz}, 10.1 \mathrm{~Hz}, 6.8 \mathrm{~Hz}, 1 \mathrm{H}), 5.05$ (ddt, $J=16.8 \mathrm{~Hz}, 2 \mathrm{~Hz}, 2 \mathrm{~Hz}, 1 \mathrm{H}$ ), 4.97 (ddt, $J=10.1 \mathrm{~Hz}, 2$ $\mathrm{Hz}, 2 \mathrm{~Hz}, 1 \mathrm{H}), 4.59$ (s, 1H), 3.46 (t, $6.5 \mathrm{~Hz}, 1 \mathrm{H}), 2.38-2.34$ (m, 2H), 2.0-1.94 (brs, 1H);

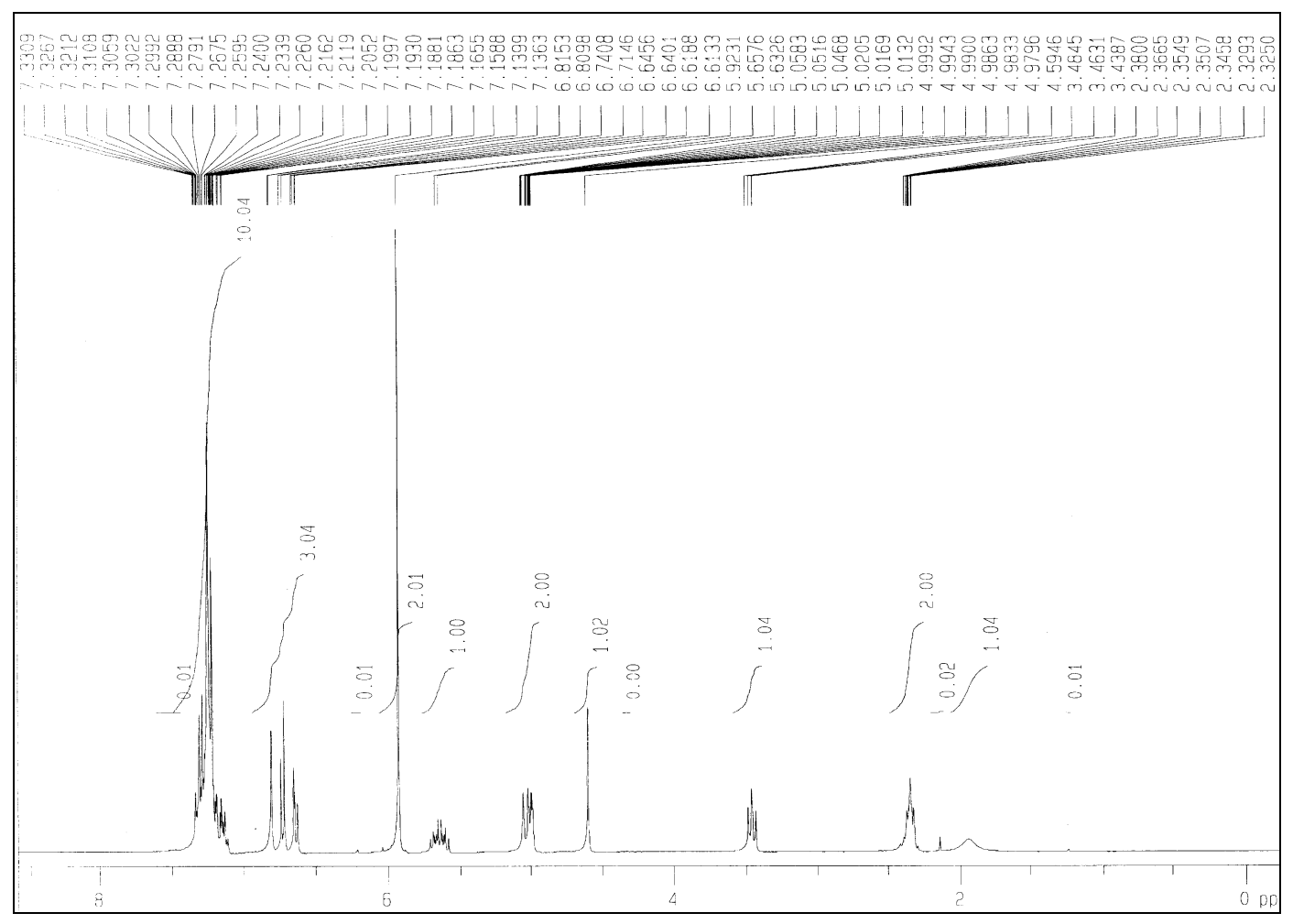

${ }^{13} \mathrm{C} \mathrm{NMR}\left(\mathrm{CDCl}_{3}\right) \delta 147.7,146.4,144.5,143.3,137.8,135.4,128.4,128.2,127.7,127.3,126.9,126.6,120.5$, 117.3, 107.9, 107.2, 100.8, 63.0, 58.9, 43.0; MS (EI) m/z (relative intensity) 316 [M+-allyl] (8.6), 167 (100), 165 (17.3), 148 (1.8), 122 (3), 115 (4.2), 76 (5.7); Anal. Calcd for $\mathrm{C}_{24} \mathrm{H}_{23} \mathrm{NO}_{2}$ (357.45): C, 80.64; H, 6.48; N, 3.92. Found: C, 80.52; H, 6.51; N, 3.8. The enantiomeric excess was determined by converting to trifluoroacetylamide form, $[\alpha]_{\mathrm{D}}^{22}=+78.2\left(\mathrm{c} 1.0, \mathrm{CHCl}_{3}\right)$ and using CHIRALCEL OD column, hexane $/ i-\mathrm{PrOH}=50 / 1$, flow rate $=0.8$ $\mathrm{mL} / \mathrm{min}$, UV detection at $254 \mathrm{~nm}, \mathrm{t}_{\mathrm{R}}=14.44 \mathrm{~min}$ (major enantiomer), $\mathrm{t}_{\mathrm{R}}=18.95 \min$ (minor enantiomer); $83 \%$ ee. 


\section{(S)-2-Allylpiperidine}

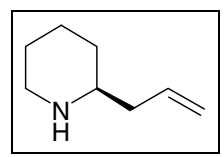

${ }^{1} \mathrm{H}$ NMR of the hydrochloride $\left(\mathrm{D}_{2} \mathrm{O}+\mathrm{CH}_{3} \mathrm{NO}_{2}\right.$ internal standard) $\delta 5.877-5.73(\mathrm{~m}, 1 \mathrm{H}), 5.26-5.2(\mathrm{~m}, 2 \mathrm{H})$, 3.39-3.35 (m, 1H), 3.17-3.13 (m, 1H), 3.06-2.87 (m, 1H), 2.52-2.29 (m, 2H), 2.00-1.43 (m, 6H);

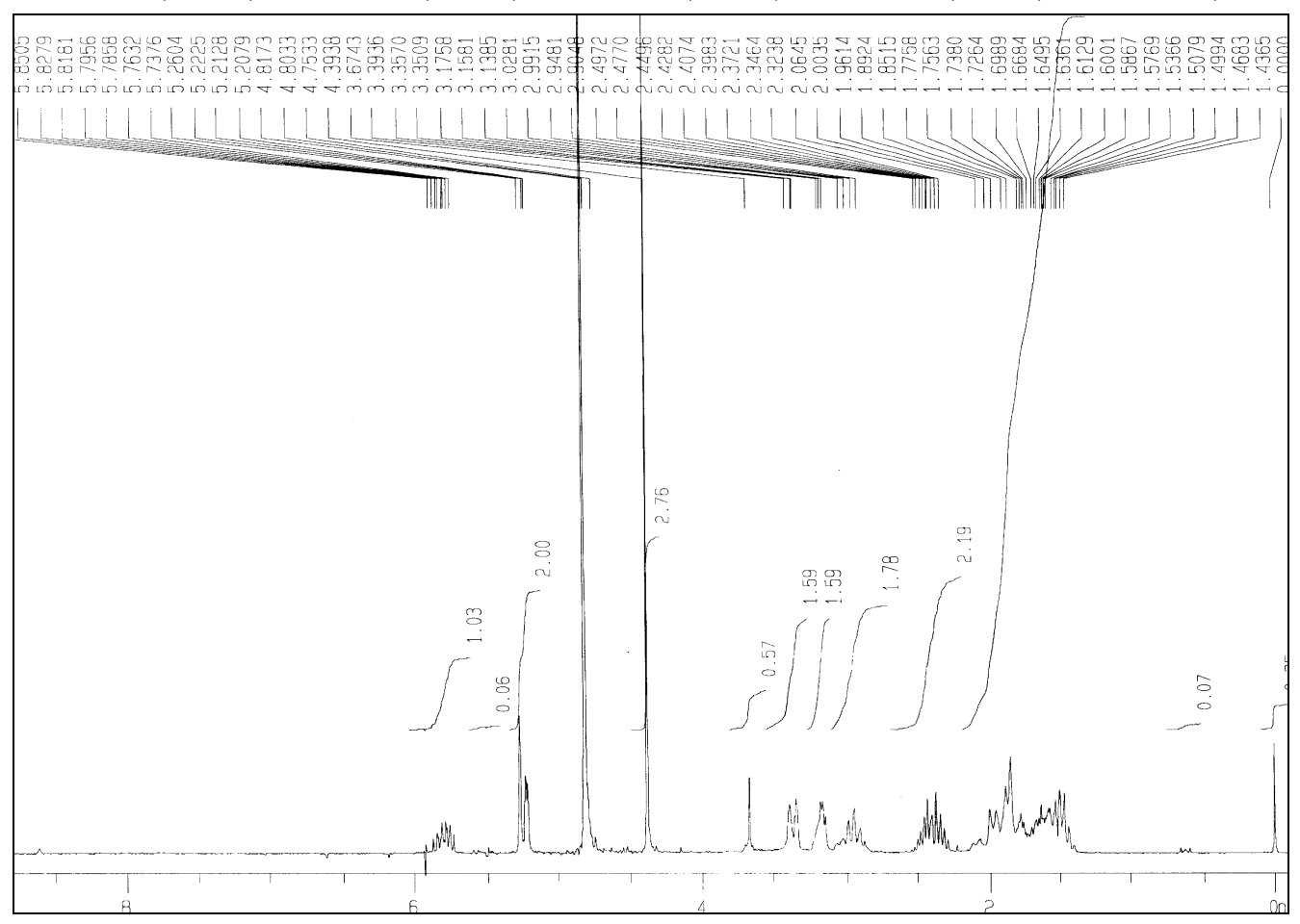

${ }^{13} \mathrm{C} \mathrm{NMR}\left(\mathrm{CDCl}_{3}\right) \delta 135.5,117.2,56.0,47.0,41.9,32.6,26.2,24.8$. The enantiomeric excess was determined by converting into $\mathrm{N}$-tosylamide, pale yellow oil; $[\alpha]_{\mathrm{D}}{ }^{21}=-0.43\left(\mathrm{c}, 0.9, \mathrm{CH}_{2} \mathrm{Cl}_{2}\right)$; and using CHIRALCEL OD column, hexane $/ i-\mathrm{PrOH}=50 / 1$, flow rate $=1.0 \mathrm{~mL} / \mathrm{min}$, UV detection at $254 \mathrm{~nm}, \mathrm{t}_{\mathrm{R}}=9.91 \mathrm{~min}$ (minor enantiomer), $t_{R}=11.12 \min$ (major enantiomer); $2 \%$ ee. Configurational assignment was made by comparison with the reported optical rotation. ${ }^{6,7}$

\section{(R)-2-Allylpiperidine}

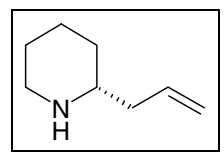

${ }^{1} \mathrm{H}$ NMR and ${ }^{13} \mathrm{C}$ NMR same as above. The enantiomeric excess was determined by converting into N-tosylamide, pale yellow oil; $[\alpha]_{\mathrm{D}}{ }^{23}=+4.1\left(\mathrm{c}, 0.995, \mathrm{CH}_{2} \mathrm{Cl}_{2}\right)$; and using CHIRALCEL OD column, hexane $/ i-\mathrm{PrOH}=50 / 1$, flow rate $=1.0 \mathrm{~mL} / \mathrm{min}, \mathrm{UV}$ detection at $254 \mathrm{~nm}, \mathrm{t}_{\mathrm{R}}=9.92 \mathrm{~min}$ (major enantiomer), $\mathrm{t}_{\mathrm{R}}=$ 11.42 min (minor enantiomer); $18 \%$ ee. 


\section{(R)-1-Allyl-1,2,3,4-tetrahydroisoquinoline}

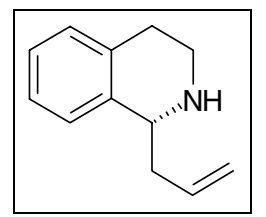

Yellow oil; $[\alpha]_{\mathrm{D}}{ }^{22}=+6.2\left(\mathrm{c} 0.61\right.$, THF); $7 \%$ ee based on $[\alpha]_{\mathrm{D}}$ reported in literature. ${ }^{6}$ Configurational assignment was made by comparison with the reported optical rotation. ${ }^{6}$ IR (neat) $3072,3018,2920,2833,2803,2728,1638$, 1492, 1454, 1430, 1315, 1132, 997, 914, $741 \mathrm{~cm}^{-1} ;{ }^{1} \mathrm{H}$ NMR $\left(\mathrm{CDCl}_{3}\right) \delta$ 7.17-7.07 (m, 4H), 5.85-5.79 (m, 1H), $5.2-5.12(\mathrm{~m}, 2 \mathrm{H}), 4.05(\mathrm{dd}, J=9.0 \mathrm{~Hz}, 3.5 \mathrm{~Hz}, 1 \mathrm{H}), 3.26-3.2(\mathrm{~m}, 1 \mathrm{H}), 3.01-2.93(\mathrm{~m}, 1 \mathrm{H}), 2.83-2.63(\mathrm{~m}, 3 \mathrm{H})$, $2.55-2.48(\mathrm{~m}, 1 \mathrm{H}), 1.81$ (brs, $1 \mathrm{H})$;

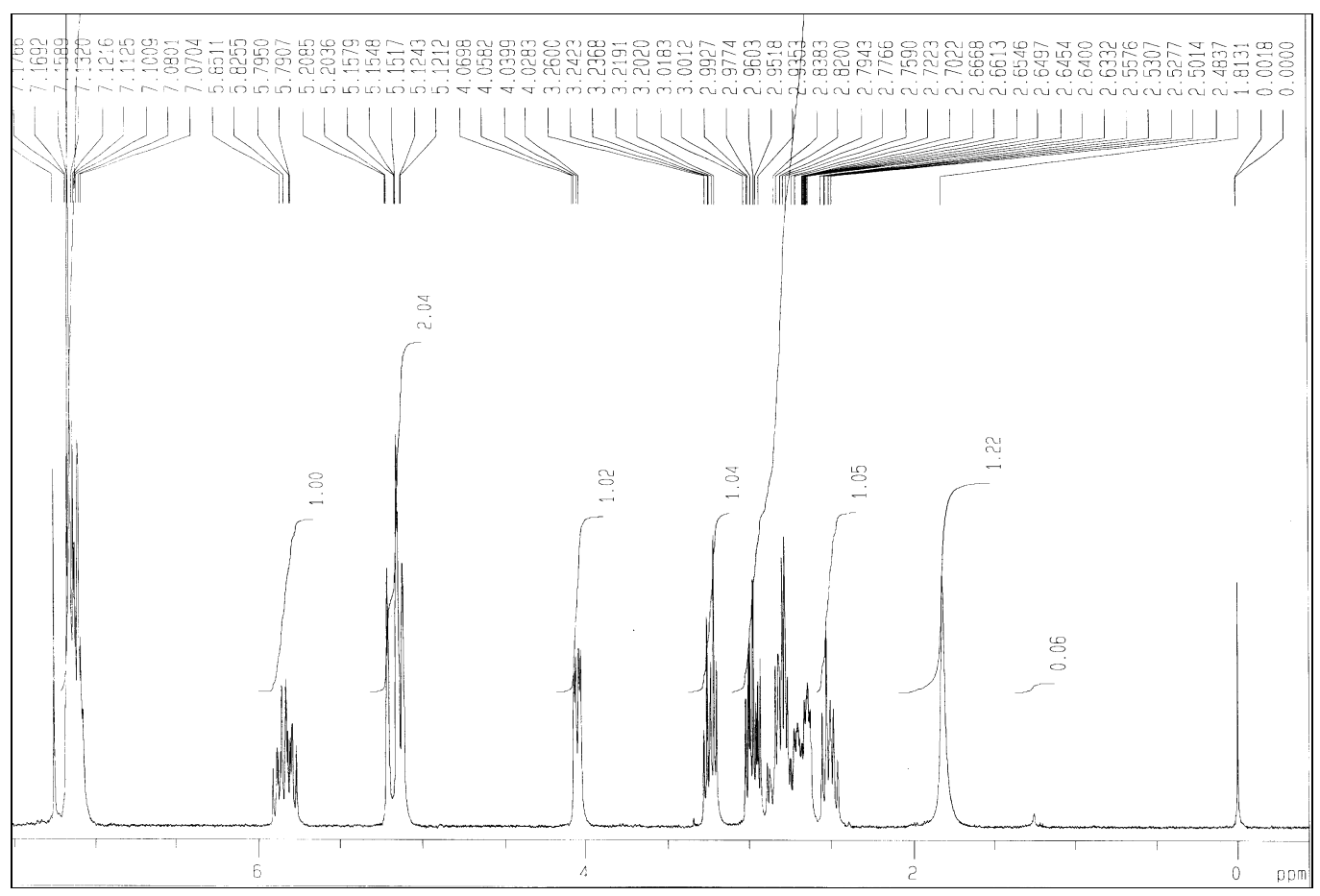

${ }^{13} \mathrm{C}$ NMR $\left(\mathrm{CDCl}_{3}\right) \delta 138.7,135.8,129.1,126.4,125.5,117.8,55.3,41.2,29.8$; MS (EI) $\mathrm{m} / \mathrm{z}$ (relative intensity) $173\left[\mathrm{M}^{+}\right](0.3), 172\left[\mathrm{M}^{+}-\mathrm{H}\right]$ (1.9), $132\left[\mathrm{M}^{+}\right.$-allyl] (100), 117 (6.9), 105 (5.1), 91 (1.2), 77 (3.5).

\section{(S)-1-Allyl-1,2,3,4-tetrahydroisoquinoline}

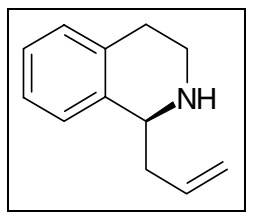

Yellow oil; $[\alpha]_{\mathrm{D}}{ }^{22}=-16.1$ (c 0.61 , THF); $19 \%$ ee based on $[\alpha]_{\mathrm{D}}$ reported in literature. ${ }^{6} \mathrm{IR},{ }^{1} \mathrm{H}$ NMR, ${ }^{13} \mathrm{C}$ NMR and MS data same as above. 


\section{References and Notes}

1. $\quad$ Yamamoto, Y.; Shimota, H.; Oda, J.; Inouye, Y. Bull. Chem. Soc. Jpn. 1976, 49, 3247.

2. $\quad$ Nakamura, H.; Nakamura, K.; Yamamoto, Y. J. Am. Chem. Soc. 1998, 120, 4242.

3. Brown, H. C.; Weissman, S. A.; Perumal, P. T.; Dhokte, U. P. J. Org. Chem. 1990, 55, 1217

4. Trost, B. M.; Strege, P. E.; Weber, L.; Fullerton, T. J.; Dietsche, T. J. J. Am. Chem. Soc. 1978, 100, 3407.

5. Trost, B. M.; Metzner, P. J. J. Am. Chem. Soc. 1980, 102, 3572.

6. (a) Nakamura, M.; Hirai, A.; Nakamura, E. J. Am. Chem. Soc. 1996, 118, 8489. (b) Meyers. A. I.; Dickman, D. A.; Boes, M. Tetrahedron 1987, 43, 5095.

7. $\quad$ Aketa, K.-I.; Terashima, S.; Yamada, S.-I. Chem, Pharm. Bull. 1976, $24,621$. 